\title{
On decay and blow-up of solutions for a nonlinear Petrovsky system with conical degeneration
}

\author{
Jiali Yu', Yadong Shang ${ }^{2^{*}}$ (D) and Huafei $\mathrm{Di}^{2}$
}

\section{"Correspondence:}

gzydshang@126.com

${ }^{2}$ School of Mathematics and

Information Science, Guangzhou

University, Guangzhou, 510006,

P.R. China

Full list of author information is

available at the end of the article

\section{Springer}

\begin{abstract}
This paper deals with a class of Petrovsky system with nonlinear damping

$$
w_{t t}+\Delta_{\mathbb{B}}^{2} w-k_{2} \Delta_{\mathbb{B}} w_{t}+a w_{t}\left|w_{t}\right|^{m-2}=b w|w|^{p-2}
$$

on a manifold with conical singularity, where $\Delta_{\mathbb{B}}$ is a Fuchsian-type Laplace operator with totally characteristic degeneracy on the boundary $x_{1}=0$. We first prove the global existence of solutions under conditions without relation between $m$ and $p$, and establish an exponential decay rate. Furthermore, we obtain a finite time blow-up result for local solutions with low initial energy $E(0)<d$.
\end{abstract}

MSC: 35B44; 35K30; 35K59

Keywords: Petrovsky system; Cone Sobolev spaces; Global existence; Decay rate; Blow-up

\section{Introduction}

Due to the frequent occurrence of high order nonlinear wave equations in many branches of engineering, physics, chemistry, material science, and other sciences, the study of wave equations plays a key role in mathematical analysis. For more details, see [1, 2]. In [3] and [4], the original Petrovsky model has the following form:

$$
\begin{aligned}
& w_{t t}+\Delta^{2} w-\Delta w_{t}+w_{t}\left|w_{t}\right|^{m-2}=w|w|^{p-2}, \quad x \in \Omega, t>0, \\
& w=0, \quad \frac{\partial w}{\partial v}=0, \quad x \in \partial \Omega, t \geq 0, \\
& w(x, 0)=w_{0}(x), \quad w_{t}(x, 0)=w_{1}(x), \quad x \in \bar{\Omega},
\end{aligned}
$$

where $\Omega \in \mathbb{R}^{n}$ is a bounded domain with a smooth boundary $\partial \Omega$.

Equation (1.1) is an important physical model that appears in many applications to mathematical physics as well as in the theory of vibrating plates, geophysics, and ocean acoustics $[5,6]$. Some further physical interpretations are given in $[7,8]$.

(c) The Author(s) 2020. This article is licensed under a Creative Commons Attribution 4.0 International License, which permits use, sharing, adaptation, distribution and reproduction in any medium or format, as long as you give appropriate credit to the original author(s) and the source, provide a link to the Creative Commons licence, and indicate if changes were made. The images or other third party material in this article are included in the article's Creative Commons licence, unless indicated otherwise in a credit line to the material. If material is not included in the article's Creative Commons licence and your intended use is not permitted by statutory regulation or exceeds the permitted use, you will need to obtain permission directly from the copyright holder. To view a copy of this licence, visit http://creativecommons.org/licenses/by/4.0/. 
For Equation (1.1), many results for global existence, nonexistence, and asymptotic behavior of solutions have been obtained [3-11]. Li et al. [3] studied problem (1.1)-(1.3) and derived that the solution is global without the relation between $m$ and $p$. Moreover, the decay estimates of the energy function and the estimates of the lifespan of solution were given. Later, under suitable conditions decay estimates of the solutions for Equation (1.1) have been established by using Nakao's inequality in [4]. Messaoudi [9] proved the solution for problem (1.1)-(1.3) without $\Delta w_{t}$ blows up in finite time if $p>m$ and the energy is negative. $\mathrm{Wu}[10]$ proved the blow-up result for problem (1.1)-(1.3) without $\Delta w_{t}$ if $p>m$ and the energy is nonnegative. Recently, Chen et al. [11] proved that the solution of problem (1.1)-(1.3) without $\Delta w_{t}$ blows up with positive initial energy and claimed that the solution blows up in finite time for even vanishing initial energy for $m=2$. More recently, Philippin et al. [12] used a differential inequality technique to obtain a lower bound on blow-up time for Equation (1.1) without $\Delta w_{t}$. In recent years, lower bounds for the blowup time in a superlinear hyperbolic equation with damping term have been derived [13]. For other related works, we refer the readers to [14-18] and the references therein.

In 2011 to 2012, Chen et al. established the corresponding Sobolev inequality on the cone Sobolev spaces in $[19,20]$. And on this basis, they studied the initial boundary value problem of a semilinear parabolic equation on a manifold with conical singularity [21] and obtained the existence and nonexistence results by introducing a family of potential wells. Li et al. [22] proved the global existence, exponential decay, and finite time blow-up of solution for a class of semilinear pseudo-parabolic equations with conical degeneration. Recently, Alimohammady et al. [23] studied a class of semilinear degenerate hyperbolic equations on the cone Sobolev spaces

$$
\begin{aligned}
& w_{t t}-\Delta_{\mathbb{B}} w+V(x) w+\gamma w_{t}=g_{t}(x) w|w|^{p-1}, \quad x \in \operatorname{int} \mathbb{B}, t>0, \\
& w(t, x)=0, \quad x \in \partial \mathbb{B}, t \geq 0, \\
& w(x, 0)=w_{0}(x), \quad w_{t}(x, 0)=w_{1}(x), \quad x \in \operatorname{int} \mathbb{B},
\end{aligned}
$$

where $\mathbb{B}=[0,1) \times X, X$ is an $(n-1)$-dimensional closed compact manifold, which is regarded as the local model near the conical points and $\partial \mathbb{B}=\{0\} \times X \cdot \Delta_{\mathbb{B}}=\left(x_{1} \partial_{x_{1}}\right)^{2}+\partial_{x_{2}}^{2}+$ $\cdots+\partial_{x_{n}}^{2}$.

They discussed the invariance of some sets, global existence, nonexistence, and asymptotic behavior of solutions with initial energy $J\left(w_{0}\right)<d$ by introducing a family of potential wells which was first proposed by Sattinger [24]. More works on equations with conical degeneration can be seen in the literature [25-28] and the references therein.

If we consider Equation (1.1) on a manifold with conical singularity, that is, when the standard Laplace operator $\Delta$ of Equation (1.1) is replaced by Fuchsian-type Laplace operator $\Delta_{\mathbb{B}}$, what will happened for the initial boundary value problem? For this kind of Petrovsky equation with conical degeneration, the existence and nonexistence of global solutions to both the initial boundary value problem and the initial value problem remain open.

Inspired by the ideas of [3, 4, 23] and [29-31], we study the initial boundary value problem for the following Petrovsky equation:

$$
w_{t t}+\Delta_{\mathbb{B}}^{2} w-k_{2} \Delta_{\mathbb{B}} w_{t}+a w_{t}\left|w_{t}\right|^{m-2}=b w|w|^{p-2}, \quad x \in \operatorname{int} \mathbb{B}, t>0
$$




$$
\begin{aligned}
& w=0, \quad \nabla_{\mathbb{B}} w \cdot v=0, \quad x \in \partial \mathbb{B}, t \geq 0, \\
& w(x, 0)=w_{0}(x), \quad w_{t}(x, 0)=w_{1}(x), \quad x \in \operatorname{int} \mathbb{B},
\end{aligned}
$$

where $w_{0}(x), w_{1}(x)$ are suitable initial data and $k_{2}, a, b, m, p$ are constants such that $k_{2}$ and $b$ are positive, $a$ is nonnegative, and $m \geq 2,2<p<\frac{2 n}{n-2}=p^{*}$, where $p^{*}$ is the critical Sobolev exponents. Here $\mathbb{B}$ is defined as above, and $v$ is the unit normal vector pointing toward the exterior of $\mathbb{B}$. Moreover, the operator $\Delta_{\mathbb{B}}$ in $(1.7)$ is defined by $\left(x_{1} \partial_{x_{1}}\right)^{2}+\partial_{x_{2}}^{2}+\cdots+\partial_{x_{n}}^{2}$, which is an elliptic operator with conical degeneration on the boundary $x_{1}=0$ (we also called it Fuchsian-type Laplace operator), and the divergence operator $\operatorname{div}_{\mathbb{B}}$ is defined by $x_{1} \partial_{x_{1}}+$ $\partial_{x_{2}}+\cdots+\partial_{x_{n}}$, the corresponding gradient operator is denoted by $\nabla_{\mathbb{B}}=\left(x_{1} \partial_{x_{1}}, \partial_{x_{2}}, \ldots, \partial_{x_{n}}\right)$. In the neighborhood of $\partial \mathbb{B}$ we will use coordinates $\left(x_{1}, x^{\prime}\right)=\left(x_{1}, x_{2}, \ldots, x_{n}\right)$ for $0 \leq x_{1}<1$, $x^{\prime} \in X$.

Our main aim in this paper is to find the existence and nonexistence of solutions for problem (1.7)-(1.9) with cone degeneration by introducing a family of potential wells. Firstly, under the condition of low initial energy, we establish the existence of global solution in the cone Sobolev space by a combination of Galerkin method and potential well theory. Then, using the energy perturbation technique, we obtain the exponential decay result of the global solution. Finally, we show that the solution of the problem blows up in a finite time and give the estimates for lower and upper bounds of blow-up time. It is worth mentioning that two types of lower bounds of the blow-up time $T_{\max }$ for the weak solution of (1.7)-(1.9) are given, respectively.

The rest of this article is organized as follows. In Sect. 2, we recall the cone Sobolev spaces and the corresponding properties. In Sect. 3, we establish a global existence result and show the decay rates. In Sect. 4, we prove the blow-up properties of local solution.

\section{Preliminaries}

In this section, we recall the manifold with conical singularities and the cone Sobolev spaces which were introduced in $[19,20]$ and introduce some lemmas and notations.

We assume that the manifold $B$ has only one conical point on the boundary. Thus, near the conical point, we have a stretched manifold $\mathbb{B}$ associated with $B$. Here $\mathbb{B}=[0,1) \times$ $X, \partial \mathbb{B}=\{0\} \times X$ and $X$ is a closed compact manifold of dimension $n-1$. Also, in the neighborhood of the conical point, we use coordinates $\left(x_{1}, x^{\prime}\right)=\left(x_{1}, x_{2}, \ldots, x_{n}\right)$ for $0 \leq x_{1}<$ $1, x^{\prime} \in X$.

Definition 2.1 Let $\mathbb{B}=[0,1) \times X$ be a stretched manifold of the manifold $B$ with conical singularity. Then the cone Sobolev space $\mathcal{H}_{p}^{m, \gamma}(\mathbb{B})$ for $m \in \mathbb{N}, \gamma \in \mathbb{R}$, and $1<p<\infty$ is defined as

$$
\mathcal{H}_{p}^{m, \gamma}(\mathbb{B})=\left\{u \in W_{\mathrm{loc}}^{m, p}(\operatorname{int} \mathbb{B}) \mid \omega u \in \mathcal{H}_{p}^{m, \gamma}\left(X^{\Lambda}\right)\right\}
$$

for any cut-off function $\omega$ supported by a collar neighborhood of $(0,1) \times \partial \mathbb{B}$. Moreover, the subspace $\mathcal{H}_{p, 0}^{m, \gamma}(\mathbb{B})$ of $\mathcal{H}_{p}^{m, \gamma}(\mathbb{B})$ is defined by

$$
\mathcal{H}_{p, 0}^{m, \gamma}(\mathbb{B})=[\omega] \mathcal{H}_{p, 0}^{m, \gamma}\left(X^{\Lambda}\right)+[1-\omega] W_{0}^{m, p}(\text { int } \mathbb{B})
$$


where $X^{\Lambda}=\mathbb{R}_{+} \times X$ as the corresponding open stretched cone with the base $X, W_{0}^{m, p}$ (int $\left.\mathbb{B}\right)$ denotes the closure of $C_{0}^{\infty}(\operatorname{int} \mathbb{B})$ in Sobolev spaces $W^{m, p}(\bar{X})$ when $\bar{X}$ is a closed compact $C^{\infty}$ manifold of dimension $n$ that contains $B$ as a submanifold with boundary.

Remark 2.1 ([32]) We have the following properties:

(1) $\mathcal{H}_{p}^{m, \gamma}(\mathbb{B})$ is a Banach space for $1 \leq p<\infty$ and is a Hilbert space for $p=2$.

(2) $L_{p}^{\gamma}(\mathbb{B}):=\mathcal{H}_{p}^{0, \gamma}(\mathbb{B})$.

(3) $L_{p}(\mathbb{B}):=\mathcal{H}_{p}^{0,0}(\mathbb{B})$.

(4) The embedding $\mathcal{H}_{p}^{m, \gamma}(\mathbb{B}) \hookrightarrow \mathcal{H}_{p}^{m^{\prime}, \gamma^{\prime}}(\mathbb{B})$ is continuous if $m \geq m^{\prime}, \gamma \geq \gamma^{\prime}$; and is compact embedding if $m>m^{\prime}, \gamma>\gamma^{\prime}$.

Definition 2.2 Let $\mathbb{B}=[0,1) \times X$. Then $u(x) \in L_{p}^{\gamma}(\mathbb{B})$ with $1<p<\infty$ and $\gamma \in \mathbb{R}$ if

$$
\|u(x)\|_{L_{p}^{\gamma}(\mathbb{B})}^{p}=\int_{\mathbb{B}} x_{1}^{n}\left|x_{1}^{-\gamma} u(x)\right|^{p} \frac{d x_{1}}{x_{1}} d x^{\prime}<+\infty .
$$

Observe that if $u(x) \in L_{p}^{\frac{n}{p}}(\mathbb{B}), v(x) \in L_{q}^{\frac{n}{q}}(\mathbb{B})$ with $p, q \in(1,+\infty)$ and $\frac{1}{p}+\frac{1}{q}=1$, then we have the following Hölder inequality:

$$
\int_{\mathbb{B}}|u(x) v(x)| \frac{d x_{1}}{x_{1}} d x^{\prime} \leq\left(\int_{\mathbb{B}}|u(x)|^{p} \frac{d x_{1}}{x_{1}} d x^{\prime}\right)^{\frac{1}{p}}\left(\int_{\mathbb{B}}|v(x)|^{q} \frac{d x_{1}}{x_{1}} d x^{\prime}\right)^{\frac{1}{q}} .
$$

In the sequel, for convenience we denote

$$
\begin{aligned}
& (u, v)_{2}=\int_{\mathbb{B}} u(x) v(x) \frac{d x_{1}}{x_{1}} d x^{\prime}, \quad\|u\|_{L_{p}^{\frac{n}{p}(\mathbb{B})}}^{p}=\int_{\mathbb{B}}|u(x)|^{p} \frac{d x_{1}}{x_{1}} d x^{\prime} . \\
& \tilde{\mathcal{H}}_{2,0}^{1, \frac{n}{2}}(\mathbb{B}):=\left\{u(x) \in \mathcal{H}_{2}^{1, \frac{n}{2}}(\mathbb{B}) \mid u=0 \text { on } \partial \mathbb{B}\right\}, \\
& \tilde{\mathcal{H}}_{2,0}^{2, \frac{n}{2}}(\mathbb{B}):=\left\{u(x) \in \mathcal{H}_{2}^{2, \frac{n}{2}}(\mathbb{B}) \mid u=\nabla_{\mathbb{B}} u \cdot v=0 \text { on } \partial \mathbb{B}\right\}, \\
& \|u\|_{\tilde{\mathcal{H}}_{2,0}^{1, \frac{n}{2}}(\mathbb{B})}^{2}=\|u\|_{L_{2}^{\frac{n}{2}(\mathbb{B})}}^{2}+\left\|\nabla_{\mathbb{B}} u\right\|_{L_{2}^{2}(\mathbb{B})}^{2}, \\
& \|u\|_{\tilde{\mathcal{H}}_{2,0}^{2, \frac{n}{2}}}^{2}=\|u\|_{L_{2}^{2}(\mathbb{B})}^{2}+\left\|\nabla_{\mathbb{B}} u\right\|_{L_{2}^{\frac{n}{2}(\mathbb{B})}}^{2}+\left\|\Delta_{\mathbb{B}} u\right\|_{L_{2}^{\frac{n}{2}(\mathbb{B})}}^{2} .
\end{aligned}
$$

The spaces $\tilde{\mathcal{H}}_{2,0}^{1, \frac{n}{2}}(\mathbb{B}), \tilde{\mathcal{H}}_{2,0}^{2, \frac{n}{2}}(\mathbb{B})$ with norms $\|u\|_{\tilde{\mathcal{H}}_{2,0}^{1, \frac{n}{2}}(\mathbb{B})},\|u\|_{\tilde{\mathcal{H}}_{2,0}^{2, \frac{n}{2}}(\mathbb{B})}$ are Banach spaces, where the norms $\|u\|_{\tilde{\mathcal{H}}_{2,0}^{1, \frac{n}{2}}(\mathbb{B})},\|u\|_{\tilde{\mathcal{H}}_{2,0}^{2, \frac{n}{2}}(\mathbb{B})}$ are equivalent to the norms $\left\|\nabla_{\mathbb{B}} u\right\|_{L_{2}^{\frac{n}{2}(\mathbb{B})}},\left\|\Delta_{\mathbb{B}} u\right\|_{L_{2}^{\frac{n}{2}(\mathbb{B})}}$, respectively.

Lemma 2.1 Let $u(x), v(x) \in \tilde{\mathcal{H}}_{2,0}^{1, \frac{n}{2}}(\mathbb{B})$. Then

$$
\int_{\mathbb{B}} v \Delta_{\mathbb{B}} u \frac{d x_{1}}{x_{1}} d x^{\prime}=-\int_{\mathbb{B}} \nabla_{\mathbb{B}} u \cdot \nabla_{\mathbb{B}} v \frac{d x_{1}}{x_{1}} d x^{\prime}
$$


Proof Here we first suppose $u(x), v(x) \in C_{0}^{\infty}(\mathbb{B})$. From the definition of $\Delta_{\mathbb{B}}$, it follows that

$$
\begin{aligned}
\int_{\mathbb{B}} v \Delta_{\mathbb{B}} u \frac{d x_{1}}{x_{1}} d x^{\prime} \\
=\int_{\mathbb{B}} x_{1} \partial_{x_{1}}\left(x_{1} \partial_{x_{1}} u\right) \cdot v \frac{d x_{1}}{x_{1}} d x^{\prime}+\int_{\mathbb{B}}\left(\partial_{x_{2}}^{2} u+\cdots+\partial_{x_{n}}^{2} u\right) \cdot v \frac{d x_{1}}{x_{1}} d x^{\prime} \\
=\int_{\mathbb{B}} \partial_{x_{1}}\left(x_{1} \partial_{x_{1}} u\right) \cdot v d x+\int_{\mathbb{B}}\left(\partial_{x_{2}}^{2} u+\cdots+\partial_{x_{n}}^{2} u\right) \cdot v \frac{d x_{1}}{x_{1}} d x^{\prime} \\
=\int_{\mathbb{B}} \operatorname{div}\left(x_{1} \partial_{x_{1}} u, \frac{\partial_{x_{2}} u}{x_{1}}, \ldots, \frac{\partial_{x_{n}} u}{x_{1}}\right) \cdot v d x \\
=-\int_{\mathbb{B}}\left(x_{1} \partial_{x_{1}} u, \frac{\partial_{x_{2}} u}{x_{1}}, \ldots, \frac{\partial_{x_{n}} u}{x_{1}}\right) \cdot \nabla v d x \\
=-\int_{\mathbb{B}}\left(x_{1}^{2} \partial_{x_{1}} u, \partial_{x_{2}} u, \ldots, \partial_{x_{n}} u\right) \cdot \nabla v \frac{d x_{1}}{x_{1}} d x^{\prime} \\
=-\int_{\mathbb{B}}\left(x_{1} \partial_{x_{1}} u, \partial_{x_{2}} u, \ldots, \partial_{x_{n}} u\right) \cdot\left(x_{1} \partial_{x_{1}} v, \partial_{x_{2}} v, \ldots, \partial_{x_{n}} v\right) \frac{d x_{1}}{x_{1}} d x^{\prime} \\
=-\int_{\mathbb{B}} \nabla_{\mathbb{B}} u \cdot \nabla_{\mathbb{B}} v \frac{d x_{1}}{x_{1}} d x^{\prime} .
\end{aligned}
$$

Finally, since $C_{0}^{\infty}(\mathbb{B})$ is dense in $\tilde{\mathcal{H}}_{2,0}^{1, \frac{n}{2}}(\mathbb{B})$, the equation above holds in the case of $u(x), v(x) \in$ $\tilde{\mathcal{H}}_{2,0}^{1, \frac{n}{2}}(\mathbb{B})$.

Lemma 2.2 ([21], Poincaré inequality) Let $\mathbb{B}=[0,1) \times X$ be a bounded subspace in $\mathbb{R}_{+}^{n}$ with $X \subset \mathbb{R}^{n-1}$, and $1<p<+\infty, \gamma \in \mathbb{R}$. If $u(x) \in \tilde{\mathcal{H}}_{p, 0}^{1, \gamma}(\mathbb{B})$, then

$$
\|u(x)\|_{L_{p}^{\gamma}(\mathbb{B})} \leq c_{\star}\left\|\nabla_{\mathbb{B}} u(x)\right\|_{L_{p}^{\gamma}(\mathbb{B})},
$$

where $\nabla_{\mathbb{B}}=\left(x_{1} \partial_{x_{1}}, \partial_{x_{2}}, \ldots, \partial_{x_{n}}\right)$ and the constant $c_{\star}$ depends only on $\mathbb{B}$.

Lemma $2.3([21])$ For $1<p<\frac{2 n}{n-2}$, the embedding $\tilde{\mathcal{H}}_{2,0}^{1, \frac{n}{2}}(\mathbb{B}) \hookrightarrow \tilde{\mathcal{H}}_{p, 0}^{0, \frac{n}{p}}(\mathbb{B})$ is continuous.

From Lemma 2.2 and Lemma 2.3, we obtain the following lemma.

Lemma 2.4 For $1<p<p^{*}$, we have

$$
\|u\|_{L_{p}^{\frac{n}{p}}(\mathbb{B})} \leq C_{0}\left\|\Delta_{\mathbb{B}} u\right\|_{L_{2}^{\frac{n}{2}}(\mathbb{B})}
$$

for $u \in \tilde{\mathcal{H}}_{2,0}^{2, \frac{n}{2}}(\mathbb{B})$ holds, where constant $C_{0}$ depends only on $\mathbb{B}$ and $p$.

\section{Global existence and energy decay}

In this section, we discuss the global existence and decay of the solution for problem (1.7)(1.9).

Similar to the classical case, we introduce the following functionals on cone Sobolev space $\tilde{\mathcal{H}}_{2,0}^{2, \frac{n}{2}}(\mathbb{B})$ :

$$
J(w)=\frac{1}{2} \int_{\mathbb{B}}\left|\Delta_{\mathbb{B}} w\right|^{2} \frac{d x_{1}}{x_{1}} d x^{\prime}-\frac{b}{p} \int_{\mathbb{B}}|w|^{p} \frac{d x_{1}}{x_{1}} d x^{\prime},
$$




$$
I(w)=\int_{\mathbb{B}}\left|\Delta_{\mathbb{B}} w\right|^{2} \frac{d x_{1}}{x_{1}} d x^{\prime}-b \int_{\mathbb{B}}|w|^{p} \frac{d x_{1}}{x_{1}} d x^{\prime}
$$

We also define the energy function as follows:

$$
E(t)=\frac{1}{2} \int_{\mathbb{B}}\left|w_{t}\right|^{2} \frac{d x_{1}}{x_{1}} d x^{\prime}+\frac{1}{2} \int_{\mathbb{B}}\left|\Delta_{\mathbb{B}} w\right|^{2} \frac{d x_{1}}{x_{1}} d x^{\prime}-\frac{b}{p} \int_{\mathbb{B}}|w|^{p} \frac{d x_{1}}{x_{1}} d x^{\prime}
$$

Finally, we introduce the potential well

$$
\left.W=\left\{w \in \tilde{\mathcal{H}}_{2,0}^{2, \frac{n}{2}}(\mathbb{B})\right) \mid I(w)>0\right\} \cup\{0\}
$$

and the outside sets of the corresponding potential well

$$
\left.V=\left\{w \in \tilde{\mathcal{H}}_{2,0}^{2, \frac{n}{2}}(\mathbb{B})\right) \mid I(w)<0\right\} .
$$

Remark 3.1 By (3.3) and Lemma 2.4, we know that

$$
E(t) \geq \frac{1}{2} \int_{\mathbb{B}}\left|\Delta_{\mathbb{B}} w\right|^{2} \frac{d x_{1}}{x_{1}} d x^{\prime}-\frac{b}{p} \int_{\mathbb{B}}|w|^{p} \frac{d x_{1}}{x_{1}} d x^{\prime} \geq g\left(\left\|\Delta_{\mathbb{B}} w\right\|_{L_{2}^{\frac{n}{2}}(\mathbb{B})}\right),
$$

where $g(\lambda)=\frac{1}{2} \lambda^{2}-\frac{b C_{0}^{p}}{p} \lambda^{p}$ and $C_{0}$ is given in Lemma 2.4. A direct calculation shows that $g(\lambda)$ has the maximum value at

$$
\lambda_{1}=\left(\frac{1}{b C_{0}^{p}}\right)^{\frac{1}{p-2}}
$$

and the maximum value is

$$
d=g\left(\lambda_{1}\right)=\frac{p-2}{2 p}\left(\frac{1}{b C_{0}^{p}}\right)^{\frac{2}{p-2}}=\frac{p-2}{2 p} \lambda_{1}^{2}>0
$$

By the definition of $g(\lambda)$ and $J(w)$, we can give another definition of $d$ as follows:

$$
d=\inf \left\{\sup _{\lambda \geq 0} J(\lambda w), w \in \tilde{\mathcal{H}}_{2,0}^{2, \frac{n}{2}}(\mathbb{B}), \int_{\mathbb{B}}\left|\Delta_{\mathbb{B}} w\right|^{2} \frac{d x_{1}}{x_{1}} d x^{\prime} \neq 0\right\}>0,
$$

and the Nehari manifold

$$
\mathcal{N}=\left\{\left.w \in \tilde{\mathcal{H}}_{2,0}^{2, \frac{n}{2}}(\mathbb{B})\left|I(w)=0, \int_{\mathbb{B}}\right| \Delta_{\mathbb{B}} w\right|^{2} \frac{d x_{1}}{x_{1}} d x^{\prime} \neq 0\right\} .
$$

Similar to the results in [29], one has $0<d=\inf _{w \in \mathcal{N}} J(w)$.

The next lemma shows that our energy functional $E(t)$ is a nonincreasing function along the solution of (1.7)-(1.9).

Lemma 3.1 $E(t)$ is a nonincreasing function for $t \geq 0$ and

$$
\frac{d}{d t} E(t)=-k_{2} \int_{\mathbb{B}}\left|\nabla_{\mathbb{B}} w_{t}\right|^{2} \frac{d x_{1}}{x_{1}} d x^{\prime}-a \int_{\mathbb{B}}\left|w_{t}\right|^{m} \frac{d x_{1}}{x_{1}} d x^{\prime} \leq 0 .
$$


Proof Multiplying (1.7) by $w_{t}$ and integrating it over $\mathbb{B} \times[0, t)$, we obtain

$$
E(t)-E(0)=-\int_{0}^{t}\left(k_{2} \int_{\mathbb{B}}\left|\nabla_{\mathbb{B}} w_{\tau}\right|^{2} \frac{d x_{1}}{x_{1}} d x^{\prime}+a \int_{\mathbb{B}}\left|w_{\tau}\right|^{m} \frac{d x_{1}}{x_{1}} d x^{\prime}\right) d \tau
$$

for $t \geq 0$. Thus, the proof is completed.

Lemma 3.2 Assume that $E(0)<d$. Then:

(i) If $\left\|\Delta_{\mathbb{B}} w_{0}\right\|_{L_{2}^{2}(\mathbb{B})}<\lambda_{1}$, then $\left\|\Delta_{\mathbb{B}} w(t)\right\|_{L_{2}^{\frac{n}{2}}(\mathbb{B})}<\lambda_{1}$ for $t \geq 0$.

(ii) If $\left\|\Delta_{\mathbb{B}} w_{0}\right\|_{L_{2}^{2}(\mathbb{B})}^{\frac{n}{2}}>\lambda_{1}$, then there exists $\lambda_{2}>\lambda_{1}$ such that $\left\|\Delta_{\mathbb{B}} w(t)\right\|_{L_{2}^{\frac{n}{2}}(\mathbb{B})} \geq \lambda_{2}$ for $t \geq 0$.

Proof From the definition of $g(\lambda)$, we see that $g(\lambda)$ is increasing in $\left(0, \lambda_{1}\right)$, decreasing in $\left(\lambda_{1}, \infty\right)$, and $g(\lambda) \rightarrow-\infty$ as $\lambda \rightarrow \infty$. Since $E(0)<d$, so there exist $\lambda_{2}$ and $\lambda_{2}^{\prime}$ such that $\lambda_{2}^{\prime}<\lambda_{1}<\lambda_{2}$ and $g\left(\lambda_{2}^{\prime}\right)=g\left(\lambda_{2}\right)=E(0)$.

(i) When $\left\|\Delta_{\mathbb{B}} w_{0}\right\|_{L_{2}^{\frac{n}{2}}(\mathbb{B})}<\lambda_{1}$, by (3.6), we have

$$
g\left(\left\|\Delta_{\mathbb{B}} w_{0}\right\|_{L_{2}^{\frac{n}{2}}(\mathbb{B})}\right) \leq E(0)=g\left(\lambda_{2}^{\prime}\right) .
$$

It implies $\left\|\Delta_{\mathbb{B}} w_{0}\right\|_{L_{2}^{\frac{n}{2}}(\mathbb{B})}<\lambda_{2}^{\prime}$. We claim that $\left\|\Delta_{\mathbb{B}} w(t)\right\|_{L_{2}^{\frac{n}{2}}(\mathbb{B})}<\lambda_{2}^{\prime}$ for $t>0$. If not, then there exists $t_{0}>0$ such that $\left\|\Delta_{\mathbb{B}} w\left(t_{0}\right)\right\|_{L_{2}^{\frac{n}{2}}(\mathbb{B})}>\lambda_{2}^{\prime}$. If $\lambda_{2}^{\prime}<\left\|\Delta_{\mathbb{B}} w\left(t_{0}\right)\right\|_{L_{2}^{\frac{n}{2}}(\mathbb{B})}<\lambda_{2}$, then $g\left(\left\|\Delta_{\mathbb{B}} w\left(t_{0}\right)\right\|_{L_{2}^{\frac{n}{2}}(\mathbb{B})}\right)>E(0) \geq E\left(t_{0}\right)$. It contradicts (3.6). If $\left\|\Delta_{\mathbb{B}} w\left(t_{0}\right)\right\|_{L_{2}^{\frac{n}{2}(\mathbb{B})}} \geq \lambda_{2}$, then by the continuity of $\left\|\Delta_{\mathbb{B}} w(t)\right\|_{L_{2}^{\frac{n}{2}}(\mathbb{B})}$, there exists $0<t_{1}<t_{0}$ such that $\lambda_{2}^{\prime}<\left\|\Delta_{\mathbb{B}} w\left(t_{1}\right)\right\|_{L_{2}^{\frac{n}{2}}(\mathbb{B})}<\lambda_{2}$, then $g\left(\left\|\Delta_{\mathbb{B}} w\left(t_{1}\right)\right\|_{L_{2}^{\frac{n}{2}}(\mathbb{B})}\right)>E(0) \geq E\left(t_{1}\right)$. This is a contradiction.

(ii) When $\left\|\Delta_{\mathbb{B}} w_{0}\right\|_{L_{2}^{\frac{n}{2}(\mathbb{B})}}>\lambda_{1}$, as in case (i) we also deduce that $\left\|\Delta_{\mathbb{B}} w_{0}\right\|_{L_{2}^{\frac{n}{2}}(\mathbb{B})}>\lambda_{1}$ implies $\left\|\Delta_{\mathbb{B}} w(t)\right\|_{L_{2}^{\frac{n}{2}}(\mathbb{B})} \geq \lambda_{2}$ for $t \geq 0$.

Lemma 3.3 Suppose that $2<p<p^{*}, w_{1} \in L_{2}^{\frac{n}{2}}(\mathbb{B})$, and $E(0)<d$. Let $w_{0} \in W$ such that

$$
\beta=b C_{0}^{p}\left(\frac{2 p}{p-2} E(0)\right)^{\frac{p-2}{2}}<1
$$

Then $w \in W$ for each $t \geq 0$.

Proof When $w=0$, we get $w \in W$ easily, so we just need to prove the case $w \neq 0$. Since $I\left(w_{0}\right)>0$, it follows from the continuity of $w$ that

$$
I(w) \geq 0
$$

for some interval near $t=0$. Let $T_{m}>0$ be a maximal time (possibly $T_{m}=T$ ) when (3.13) holds on $\left[0, T_{m}\right)$.

From (3.1)-(3.2), it follows that

$$
\begin{aligned}
J(w) & =\frac{p-2}{2 p} \int_{\mathbb{B}}\left|\Delta_{\mathbb{B}} w\right|^{2} \frac{d x_{1}}{x_{1}} d x^{\prime}+\frac{1}{p} I(w) \\
& \geq \frac{p-2}{2 p} \int_{\mathbb{B}}\left|\Delta_{\mathbb{B}} w\right|^{2} \frac{d x_{1}}{x_{1}} d x^{\prime}, \quad \text { on } t \in\left[0, T_{m}\right) .
\end{aligned}
$$


By using (3.14), (3.3), and Lemma 3.1, we get

$$
\begin{aligned}
\int_{\mathbb{B}}\left|\Delta_{\mathbb{B}} w\right|^{2} \frac{d x_{1}}{x_{1}} d x^{\prime} & \leq \frac{2 p}{p-2} J(w) \\
& \leq \frac{2 p}{p-2} E(t) \\
& \leq \frac{2 p}{p-2} E(0) .
\end{aligned}
$$

Then, by Lemma 2.4 and (3.15), we obtain

$$
\begin{aligned}
b\|w\|_{L_{p}^{\frac{n}{p}}(\mathbb{B})}^{p} & \leq b C_{0}^{p}\left\|\Delta_{\mathbb{B}} w\right\|_{L_{2}^{\frac{n}{2}}(\mathbb{B})}^{p} \\
& \leq b C_{0}^{p}\left(\frac{2 p}{p-2} E(0)\right)^{\frac{p-2}{2}}\left\|\Delta_{\mathbb{B}} w\right\|_{L_{2}^{\frac{n}{2}}(\mathbb{B})}^{2} \\
& =\beta\left\|\Delta_{\mathbb{B}} w\right\|_{L_{2}^{\frac{n}{2}}(\mathbb{B})}^{2} \\
& <\left\|\Delta_{\mathbb{B}} w\right\|_{L_{2}^{\frac{n}{2}}(\mathbb{B})}^{2}
\end{aligned}
$$

on $t \in\left[0, T_{m}\right)$. Therefore, by using (3.2), we conclude that $I(w)>0$ for all $t \in\left[0, T_{m}\right)$. By repeating the procedure, $T_{m}$ is extended to $T$. The proof is completed.

Remark 3.2 From Lemma 3.3, we can deduce that

$$
\left\|\Delta_{\mathbb{B}} w\right\|_{L_{2}^{\frac{n}{2}(\mathbb{B})}}^{2} \leq \frac{1}{1-\beta} I(w) .
$$

Theorem 3.1 Suppose that $2<p<p^{\star}, w_{1} \in L_{2}^{\frac{n}{2}}(\mathbb{B})$, and $E(0)<d$, let $w_{0} \in W$ and $w$ satisfy the assumption of Lemma 3.3. Then problem (1.7)-(1.9) admits a global weak solution $w(x, t) \in L^{\infty}\left([0, T] ; \tilde{\mathcal{H}}_{2,0}^{2, \frac{n}{2}}(\mathbb{B})\right)$ with $w_{t}(x, t) \in L^{2}\left([0, T] ; \tilde{\mathcal{H}}_{2,0}^{1, \frac{n}{2}}(\mathbb{B})\right) \cap L^{m}\left([0, T] ; L_{m}^{\frac{n}{m}}(\mathbb{B})\right) \cap$ $L^{\infty}\left([0, T] ; L_{2}^{\frac{n}{2}}(\mathbb{B})\right)$. Moreover, $w(t) \in W$ for $0 \leq t<\infty$.

Proof Let $\left\{\omega_{j}(x)\right\}$ be a system of base functions in $\tilde{\mathcal{H}}_{2,0}^{2, \frac{n}{2}}(\mathbb{B})$. Now we construct the following approximate solution $w_{s}(x, t)$ of problem (1.7)-(1.9):

$$
w_{s}(x, t)=\sum_{j=1}^{s} g_{j s}(t) \omega_{j}(x), \quad s=1,2, \ldots
$$

which satisfies

$$
\begin{aligned}
& \left(w_{s t t}, \omega_{j}\right)_{2}+\left(\Delta_{\mathbb{B}} w_{s}, \Delta_{\mathbb{B}} \omega_{j}\right)_{2}+k_{2}\left(\nabla_{\mathbb{B}} w_{s t}, \nabla_{\mathbb{B}} \omega_{j}\right)_{2}+a\left(w_{s t}\left|u_{s t}\right|^{m-2}, \omega_{j}\right)_{2} \\
& \quad=b\left(w_{s}\left|u_{s}\right|^{p-2}, \omega_{j}\right)_{2}, \quad s=1,2, \ldots, \\
& w_{s}(x, 0)=\sum_{j=1}^{s} g_{j s}(0) \omega_{j}(x) \rightarrow w_{0}(x) \quad \text { in } \tilde{\mathcal{H}}_{2,0}^{2, \frac{n}{2}}(\mathbb{B}), \\
& w_{s t}(x, 0)=\sum_{j=1}^{s} g_{j s}^{\prime}(0) \omega_{j}(x) \rightarrow w_{1}(x) \quad \text { in } L_{2}^{\frac{n}{2}}(\mathbb{B}) .
\end{aligned}
$$


Multiplying (3.18) by $g_{j s}^{\prime}(t)$, summing for $j(j=1,2, \ldots, s)$, and integrating from 0 to $t$, we obtain

$$
\begin{aligned}
& k_{2} \int_{0}^{t}\left\|\nabla_{\mathbb{B}} w_{s \tau}\right\|_{L_{2}^{\frac{n}{2}}(\mathbb{B})}^{2} d \tau+a \int_{0}^{t}\left\|w_{s \tau}\right\|_{L_{m}^{\frac{n}{m}}}^{m} d \tau+E\left(w_{s}(t)\right) \\
& \quad=E\left(w_{s}(0)\right), \quad 0 \leq t<\infty .
\end{aligned}
$$

By (3.19) we can get $E\left(w_{s}(0)\right) \rightarrow E\left(w_{0}\right)$, then for sufficiently large $m$, we have

$$
\begin{aligned}
& k_{2} \int_{0}^{t}\left\|\nabla_{\mathbb{B}} w_{s \tau}\right\|_{L_{2}^{\frac{n}{2}}(\mathbb{B})}^{2} d \tau+a \int_{0}^{t}\left\|w_{s \tau}\right\|_{L_{m}^{\frac{n}{m}}(\mathbb{B})}^{m} d \tau+E\left(w_{s}(t)\right)<d, \\
& \quad 0 \leq t<\infty
\end{aligned}
$$

From (3.22) and the proof of Lemma 3.3, we can get $w_{s}(t) \in W$ for $0 \leq t<\infty$ and sufficiently large $s$. Hence, by (3.22) and

$$
E\left(w_{s}\right)=\frac{1}{2}\left\|w_{s t}\right\|_{L_{2}^{\frac{n}{2}}(\mathbb{B})}^{2}+\frac{p-2}{2 p}\left\|\Delta_{\mathbb{B}} w_{s}\right\|_{L_{2}^{\frac{n}{2}}(\mathbb{B})}^{2}+\frac{1}{p} I\left(w_{s}\right),
$$

we obtain

$$
\begin{aligned}
& k_{2} \int_{0}^{t}\left\|\nabla_{\mathbb{B}} w_{s \tau}\right\|_{L_{2}^{\frac{n}{2}}(\mathbb{B})}^{2} d \tau+a \int_{0}^{t}\left\|w_{s \tau}\right\|_{L_{m}^{\frac{n}{m}}(\mathbb{B})}^{m} d \tau+\frac{1}{2}\left\|w_{s t}\right\|_{L_{2}^{\frac{n}{2}}(\mathbb{B})}^{2} \\
& +\frac{p-2}{2 p}\left\|\Delta_{\mathbb{B}} w_{s}\right\|_{L_{2}^{\frac{n}{2}}(\mathbb{B})}^{2}<d, \quad 0 \leq t<\infty,
\end{aligned}
$$

for sufficiently large $s$, which yields

$$
\begin{aligned}
& \left\|\Delta_{\mathbb{B}} w_{s}\right\|_{L_{2}^{\frac{n}{2}}(\mathbb{B})}^{2}<\frac{2 p}{p-2} d, \quad 0 \leq t<\infty \\
& \int_{0}^{t}\left\|\nabla_{\mathbb{B}} w_{s \tau}\right\|_{L_{2}^{\frac{n}{2}}(\mathbb{B})}^{2} d \tau<\frac{d}{k_{2}}, \quad 0 \leq t<\infty,
\end{aligned}
$$

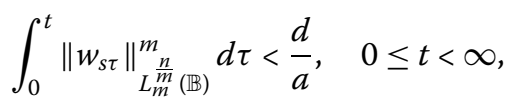

$$
\begin{aligned}
& \left\|w_{s t}\right\|_{L_{2}^{\frac{n}{2}}}^{2}<2 d, \quad 0 \leq t<\infty, \\
& \left.\left.\int_{\mathbb{B}}|| w_{s}\right|^{p-2} w_{s}\right|^{\frac{p}{p-1}} \frac{d x_{1}}{x_{1}} d x^{\prime}=\int_{\mathbb{B}}\left|w_{s}\right|^{p} \frac{d x_{1}}{x_{1}} d x^{\prime}=\left\|w_{s}\right\|_{L_{p}^{\frac{n}{p}}(\mathbb{B})}^{p} \\
& \leq C_{0}^{p}\left\|\Delta_{\mathbb{B}} w_{s}\right\|_{L_{2}^{\frac{n}{2}(\mathbb{B})}}^{p} \leq C_{0}^{p}\left(\frac{2 p}{p-2} d\right)^{\frac{p}{2}}, \\
& \left.\left.\int_{0}^{t} \int_{\mathbb{B}}|| w_{s \tau}\right|^{m-2} w_{s \tau}\right|^{\frac{m}{m-1}} \frac{d x_{1}}{x_{1}} d x^{\prime} d \tau \\
& =\int_{0}^{t} \int_{\mathbb{B}}\left|w_{s \tau}\right|^{m} \frac{d x_{1}}{x_{1}} d x^{\prime} d \tau=\int_{0}^{t}\left\|w_{s \tau}\right\|_{L_{m}^{\frac{n}{m}}(\mathbb{B})}^{m} d \tau<\frac{d}{a} .
\end{aligned}
$$


Therefore, there exist $w$ and a subsequence still denoted by $\left\{w_{s}\right\}$ for which, as $s \rightarrow \infty$,

$$
\begin{aligned}
& w_{s} \rightarrow w \quad \text { in } L^{\infty}\left(0, \infty ; \tilde{\mathcal{H}}_{2,0}^{2, \frac{n}{2}}(\mathbb{B})\right) \text { weakly star and a.e. in int } \mathbb{B} \times[0, \infty), \\
& w_{s t} \rightarrow w_{t} \quad \text { in } L^{2}\left(0, \infty ; \tilde{\mathcal{H}}_{2,0}^{1, \frac{n}{2}}(\mathbb{B})\right) \text { weakly, } \\
& w_{s t} \rightarrow w_{t} \quad \text { in } L^{m}\left(0, \infty ; L_{m}^{\frac{n}{m}}(\mathbb{B})\right) \text { weakly, } \\
& w_{s t} \rightarrow w_{t} \quad \text { in } L^{\infty}\left(0, \infty ; L_{2}^{\frac{n}{2}}(\mathbb{B})\right) \text { weakly star, } \\
& w_{s}^{p-1} \rightarrow w^{p-1} \quad \text { in } L^{\infty}\left(0, \infty ; L_{\frac{p}{p-1}}^{\frac{n(p-1)}{p}}(\mathbb{B})\right) \text { weakly star, } \\
& w_{s t}^{m-1} \rightarrow w_{t}^{m-1} \quad \text { in } L^{\frac{m}{m-1}}\left(0, \infty ; L_{\frac{n(m-1)}{m-1}}^{\frac{m}{m-1}}(\mathbb{B})\right) \text { weakly. }
\end{aligned}
$$

In (3.18), we fix $j$, letting $s \rightarrow \infty$ and integrating from 0 to $t$. Then we have

$$
\begin{aligned}
& \left(w_{t}, \omega_{j}\right)_{2}+\int_{0}^{t}\left(\Delta_{\mathbb{B}} w, \Delta_{\mathbb{B}} \omega_{j}\right)_{2} d \tau+k_{2} \int_{0}^{t}\left(\nabla_{\mathbb{B}} w_{\tau}, \nabla_{\mathbb{B}} \omega_{j}\right)_{2} d \tau \\
& +a \int_{0}^{t}\left(w_{\tau}\left|u_{\tau}\right|^{m-2}, \omega_{j}\right)_{2} d \tau=b \int_{0}^{t}\left(w|w|^{p-2}, \omega_{j}\right)_{2} d \tau+\left(w_{1}, \omega_{j}\right)_{2}
\end{aligned}
$$

and

$$
\begin{aligned}
& \left(w_{t}, v\right)_{2}+\int_{0}^{t}\left(\Delta_{\mathbb{B}} w, \Delta_{\mathbb{B}} v\right)_{2} d \tau+k_{2} \int_{0}^{t}\left(\nabla_{\mathbb{B}} w_{\tau}, \nabla_{\mathbb{B}} v\right)_{2} d \tau \\
& \quad+a \int_{0}^{t}\left(w_{\tau}\left|u_{\tau}\right|^{m-2}, v\right)_{2} d \tau=b \int_{0}^{t}\left(w|w|^{p-2}, v\right)_{2} d \tau+\left(w_{1}, v\right)_{2} \\
& \quad \forall v \in \tilde{\mathcal{H}}_{2,0}^{2, \frac{n}{2}}(\mathbb{B}) .
\end{aligned}
$$

From (3.19) we obtain $w(x, 0)=w_{0}(x)$ in $\tilde{\mathcal{H}}_{2,0}^{2, \frac{n}{2}}(\mathbb{B})$ and $w_{t}(x, 0)=w_{1}(x)$ in $L_{2}^{\frac{n}{2}}(\mathbb{B}), t \in$ $(0, T)$. By density we obtain $w \in L^{\infty}\left([0, T] ; \tilde{\mathcal{H}}_{2,0}^{2, \frac{n}{2}}(\mathbb{B})\right)$ with $w_{t} \in L^{2}\left([0, T] ; \tilde{\mathcal{H}}_{2,0}^{1, \frac{n}{2}}(\mathbb{B})\right) \cap$ $L^{m}\left([0, T] ; L_{m}^{\frac{n}{m}}(\mathbb{B})\right) \cap L^{\infty}\left([0, T] ; L_{2}^{\frac{n}{2}}(\mathbb{B})\right)$ is a global weak solution of problem (1.7)-(1.9). It is obvious that $w(t) \in W$ for $0 \leq t<\infty$.

Now, we use the following "modified" functional:

$$
G(t)=E(t)+\varepsilon\left(\int_{\mathbb{B}} w w_{t} \frac{d x_{1}}{x_{1}} d x^{\prime}+\frac{k_{2}}{2}\left\|\nabla_{\mathbb{B}} w\right\|_{L_{2}^{\frac{n}{2}}(\mathbb{B})}^{2}\right) .
$$

Lemma 3.4 Let $w$ satisfy the assumption of Theorem 3.1. For $\varepsilon$ small enough, we have

$$
\alpha_{1} G(t) \leq E(t) \leq \alpha_{2} G(t)
$$

holds for two positive constants $\alpha_{1}$ and $\alpha_{2}$. 
Proof Making use of (3.23), straightforward computations lead to

$$
\begin{aligned}
G(t)= & E(t)+\varepsilon\left(\int_{\mathbb{B}} w w_{t} \frac{d x_{1}}{x_{1}} d x^{\prime}+\frac{k_{2}}{2} \int_{\mathbb{B}}\left|\nabla_{\mathbb{B}} w\right|^{2} \frac{d x_{1}}{x_{1}} d x^{\prime}\right) \\
\leq & E(t)+\frac{\varepsilon}{2} \int_{\mathbb{B}}|w|^{2} \frac{d x_{1}}{x_{1}} d x^{\prime}+\frac{\varepsilon}{2} \int_{\mathbb{B}}\left|w_{t}\right|^{2} \frac{d x_{1}}{x_{1}} d x^{\prime} \\
& +\frac{\varepsilon k_{2}}{2} \int_{\mathbb{B}}\left|\nabla_{\mathbb{B}} w\right|^{2} \frac{d x_{1}}{x_{1}} d x^{\prime} \\
\leq & E(t)+\frac{\varepsilon}{2}\left(k_{2}+c_{\star}^{2}\right) \int_{\mathbb{B}}\left|\nabla_{\mathbb{B}} w\right|^{2} \frac{d x_{1}}{x_{1}} d x^{\prime}+\frac{\varepsilon}{2} \int_{\mathbb{B}}\left|w_{t}\right|^{2} \frac{d x_{1}}{x_{1}} d x^{\prime} \\
\leq & E(t)+\frac{\varepsilon}{2} C_{1} \int_{\mathbb{B}}\left|\Delta_{\mathbb{B}} w\right|^{2} \frac{d x_{1}}{x_{1}} d x^{\prime}+\frac{\varepsilon}{2} \int_{\mathbb{B}}\left|w_{t}\right|^{2} \frac{d x_{1}}{x_{1}} d x^{\prime} \\
\leq & E(t)+\frac{\varepsilon}{2} \frac{2 p C_{1}}{p-2} E(t)+\varepsilon E(t) \\
\leq & \frac{1}{\alpha_{1}} E(t),
\end{aligned}
$$

and in the same way, we get

$$
\begin{aligned}
G(t) & \geq E(t)-\frac{\varepsilon}{2} C_{1} \int_{\mathbb{B}}\left|\Delta_{\mathbb{B}} w\right|^{2} \frac{d x_{1}}{x_{1}} d x^{\prime}-\frac{\varepsilon}{2} \int_{\mathbb{B}}\left|w_{t}\right|^{2} \frac{d x_{1}}{x_{1}} d x^{\prime} \\
& \geq E(t)-\frac{\varepsilon}{2} \frac{2 p C_{1}}{p-2} E(t)-\varepsilon E(t) \\
& \geq \frac{1}{\alpha_{2}} E(t)
\end{aligned}
$$

for $\varepsilon$ small enough.

Theorem 3.2 Suppose that $2 \leq m<m^{*}=\frac{n-2}{2 n}$. Let $w(x, t)$ satisfy the assumption of Theorem 3.1. Then we have the following decay estimates:

$$
E(t) \leq K e^{-k t}, \quad t \geq 0
$$

where $K$ and $k$ are positive constants which will be defined later.

Proof From the definition of $G(t)$, we get

$$
\begin{aligned}
G^{\prime}(t)= & -k_{2} \int_{\mathbb{B}}\left|\nabla_{\mathbb{B}} w_{t}\right|^{2} \frac{d x_{1}}{x_{1}} d x^{\prime}-a \int_{\mathbb{B}}\left|w_{t}\right|^{m} \frac{d x_{1}}{x_{1}} d x^{\prime}+\varepsilon \int_{\mathbb{B}}\left|w_{t}\right|^{2} \frac{d x_{1}}{x_{1}} d x^{\prime} \\
& +\varepsilon \int_{\mathbb{B}} w\left(w_{t t}-k_{2} \Delta_{\mathbb{B}} w_{t}\right) \frac{d x_{1}}{x_{1}} d x^{\prime} \\
= & -k_{2} \int_{\mathbb{B}}\left|\nabla_{\mathbb{B}} w_{t}\right|^{2} \frac{d x_{1}}{x_{1}} d x^{\prime}-a \int_{\mathbb{B}}\left|w_{t}\right|^{m} \frac{d x_{1}}{x_{1}} d x^{\prime}+\varepsilon \int_{\mathbb{B}}\left|w_{t}\right|^{2} \frac{d x_{1}}{x_{1}} d x^{\prime} \\
& +\varepsilon \int_{\mathbb{B}} b|w|^{p} \frac{d x_{1}}{x_{1}} d x^{\prime}-\varepsilon \int_{\mathbb{B}} a w w_{t}\left|u_{t}\right|^{m-2} \frac{d x_{1}}{x_{1}} d x^{\prime} \\
& -\varepsilon \int_{\mathbb{B}} w \Delta_{\mathbb{B}}^{2} w \frac{d x_{1}}{x_{1}} d x^{\prime}
\end{aligned}
$$




$$
\begin{aligned}
= & -k_{2}\left\|\nabla_{\mathbb{B}} w_{t}\right\|_{L_{2}^{\frac{n}{2}(\mathbb{B})}}^{2}-a\left\|w_{t}\right\|_{L_{m}^{\frac{n}{m}(\mathbb{B})}}^{m}+\varepsilon\left\|w_{t}\right\|_{L_{2}^{\frac{2}{2}}(\mathbb{B})}^{2}+\varepsilon\left(\frac{p}{2}\left\|w_{t}\right\|_{L_{2}^{\frac{n}{2}}(\mathbb{B})}^{2}\right. \\
& \left.+\frac{p}{2}\left\|\Delta_{\mathbb{B}} w\right\|_{L_{2}^{\frac{n}{2}(\mathbb{B})}}^{2}-p E(t)\right)-\varepsilon \int_{\mathbb{B}} a w w_{t}\left|w_{t}\right|^{m-2} \frac{d x_{1}}{x_{1}} d x^{\prime} \\
& -\varepsilon\left\|\Delta_{\mathbb{B}} w\right\|_{L_{2}^{\frac{n}{2}(\mathbb{B})}}^{2} \\
= & -k_{2}\left\|\nabla_{\mathbb{B}} w_{t}\right\|_{L_{2}^{\frac{n}{2}(\mathbb{B})}}^{2}-a\left\|w_{t}\right\|_{L_{m}^{\frac{n}{m}}(\mathbb{B})}^{m}+\varepsilon\left(\frac{p}{2}+1\right)\left\|w_{t}\right\|_{L_{2}^{\frac{n}{2}}(\mathbb{B})}^{2} \\
& +\varepsilon\left(\frac{p}{2}-1\right)\left\|\Delta_{\mathbb{B}} w\right\|_{L_{2}^{2}}^{2}-\varepsilon p E(t) \\
& -\varepsilon \int_{\mathbb{B}} a w w_{t}\left|w_{t}\right|^{m-2} \frac{d x_{1}}{x_{1}} d x^{\prime} .
\end{aligned}
$$

Using Lemma 2.2, we obtain

$$
\begin{aligned}
G^{\prime}(t) \leq & {\left[\varepsilon\left(\frac{p}{2}+1\right) c_{\star}^{2}-k_{2}\right]\left\|\nabla_{\mathbb{B}} w_{t}\right\|_{L_{2}^{\frac{n}{2}(\mathbb{B})}}^{2}-a\left\|w_{t}\right\|_{L_{m}^{\frac{n}{m}(\mathbb{B})}}^{m} } \\
& +\left(\frac{p}{2}-1\right) \varepsilon\left\|\Delta_{\mathbb{B}} w\right\|_{L_{2}^{\frac{n}{2}(\mathbb{B})}}^{2}-\varepsilon p E(t) \\
& -\varepsilon a \int_{\mathbb{B}} w w_{t}\left|u_{t}\right|^{m-2} \frac{d x_{1}}{x_{1}} d x^{\prime} .
\end{aligned}
$$

Then, we will show that from the estimate of the last term in (3.38), by Young's inequality and the proof of Lemma 3.3, we obtain

$$
\begin{aligned}
& \left.\left|\int_{\mathbb{B}} w w_{t}\right| u_{t}\right|^{m-2} \frac{d x_{1}}{x_{1}} d x^{\prime} \mid \leq \theta\left\|w_{t}\right\|_{L_{m}^{\frac{n}{m}(\mathbb{B})}}^{m}+c(\theta)\|w\|_{L_{m}^{\frac{n}{m}}(\mathbb{B})}^{m}, \\
& \|w\|_{L_{m}^{\frac{n}{m}}}^{m} \leq C_{0}^{m}\left(\frac{2 p}{p-2} E(0)\right)^{\frac{m-2}{2}}\left\|\Delta_{\mathbb{B}} w\right\|_{L_{2}^{\frac{n}{2}}(\mathbb{B})}^{2} .
\end{aligned}
$$

Then by exploiting (3.38)-(3.40), we arrive at

$$
\begin{aligned}
G^{\prime}(t) \leq & {\left[\varepsilon\left(\frac{p}{2}+1\right) c_{\star}^{2}-k_{2}\right]\left\|\nabla_{\mathbb{B}} w_{t}\right\|_{L_{2}^{\frac{n}{2}}(\mathbb{B})}^{2}+(\varepsilon \theta-1) a\left\|w_{t}\right\|_{L_{m}^{\frac{n}{m}}}^{m}(\mathbb{B}) } \\
& +\varepsilon\left[a c(\theta) C_{0}^{m}\left(\frac{2 p}{p-2} E(0)\right)^{\frac{m-2}{2}}+\left(\frac{p}{2}-1\right)\right]\left\|\Delta_{\mathbb{B}} w\right\|_{L_{2}^{\frac{n}{2}}(\mathbb{B})}^{2} \\
& -\varepsilon p E(t) \\
\leq & {\left[\varepsilon\left(\frac{p}{2}+1\right) c_{\star}^{2}-k_{2}\right]\left\|\nabla_{\mathbb{B}} w_{t}\right\|_{L_{2}^{\frac{n}{2}}(\mathbb{B})}^{2}+(\varepsilon \theta-1) a\left\|w_{t}\right\|_{L_{m}^{\frac{n}{m}}(\mathbb{B})}^{m} } \\
& +\varepsilon\left[a c(\theta) C_{0}^{m}\left(\frac{2 p}{p-2} E(0)\right)^{\frac{m-2}{2}}+\left(\frac{p}{2}-1\right)\right] E(t)-\varepsilon p E(t) \\
= & {\left[\varepsilon\left(\frac{p}{2}+1\right) c_{\star}^{2}-k_{2}\right]\left\|\nabla_{\mathbb{B}} w_{t}\right\|_{L_{2}^{\frac{n}{2}}(\mathbb{B})}^{2}+(\varepsilon \theta-1) a\left\|w_{t}\right\|_{L_{m}^{\frac{n}{m}}}^{m}(\mathbb{B}) } \\
& -\varepsilon\left\{p-\left[a c(\theta) C_{0}^{m}\left(\frac{2 p}{p-2} E(0)\right)^{\frac{m-2}{2}}+\left(\frac{p}{2}-1\right)\right]\right\} E(t) .
\end{aligned}
$$


Choose $\varepsilon$ so small that $\varepsilon\left(\frac{p}{2}+1\right) c_{\star}^{2}-k_{2} \leq 0, \varepsilon \theta-1 \leq 0$. And choose suitable $\theta$ such that $p-\left[a c(\theta) C_{0}^{m}\left(\frac{2 p}{p-2} E(0)\right)^{\frac{m-2}{2}}+\left(\frac{p}{2}-1\right)\right]>0$. Then from the above inequality, we obtain

$$
G^{\prime}(t) \leq-\varepsilon\left\{p-\left[a c(\theta) C_{0}^{m}\left(\frac{2 p}{p-2} E(0)\right)^{\frac{m-2}{2}}+\left(\frac{p}{2}-1\right)\right]\right\} E(t) .
$$

Then, by the relation between $E(t)$ and $G(t)$, we get

$$
G^{\prime}(t) \leq-\varepsilon \alpha_{1}\left\{p-\left[a c(\theta) C_{0}^{m}\left(\frac{2 p}{p-2} E(0)\right)^{\frac{m-2}{2}}+\left(\frac{p}{2}-1\right)\right]\right\} G(t)
$$

We take $\varepsilon$ small enough such that

$$
G(0)=E(0)+\varepsilon\left(\int_{\mathbb{B}} w_{0} w_{1} \frac{d x_{1}}{x_{1}} d x^{\prime}+\frac{k_{2}}{2}\left\|\nabla_{\mathbb{B}} w_{0}\right\|_{L_{2}^{\frac{n}{2}}}^{2}\right)>0
$$

Integrating (3.43), we obtain

$$
G(t) \leq G(0) e^{-k t}, \quad t \geq 0,
$$

where

$$
k=\varepsilon \alpha_{1}\left\{p-\left[a c(\theta) C_{0}^{m}\left(\frac{2 p}{p-2} E(0)\right)^{\frac{m-2}{2}}+\left(\frac{p}{2}-1\right)\right]\right\}>0 .
$$

By using (3.33) again, we get

$$
E(t) \leq K e^{-k t}, \quad t \geq 0
$$

where $K=\alpha_{2} G(0)$. This completes the proof.

\section{Finite time blow-up of solution}

In this section, we show that the solution of problem (1.7)-(1.9) blows up in finite time if $p>m$ and $E(0)<d$. For this purpose, we first give the following lemma which will be used later.

Lemma 4.1 Suppose that $2<p<p^{\star}, E(0)<d, w_{1} \in L_{2}^{\frac{n}{2}}(\mathbb{B})$. Let $w_{0} \in V$, then we have

$$
\begin{aligned}
& w(t) \in V, \quad \forall t \in[0, T), \\
& d<\frac{p-2}{2 p}\left\|\Delta_{\mathbb{B}} w\right\|_{L_{2}^{\frac{n}{2}}(\mathbb{B})}^{2}, \quad \forall t \in[0, T) .
\end{aligned}
$$

Proof Let $w_{0} \in V$, we have to prove that $w(t) \in V$ for all $t \in[0, T)$. We argue by contradiction. Assume that there exists $t_{0} \in[0, T)$ such that $w\left(t_{0}\right) \notin V$. This implies that

$$
\left\|\Delta_{\mathbb{B}} w\left(t_{0}\right)\right\|_{L_{2}^{\frac{n}{2}}(\mathbb{B})}^{2} \geq b\left\|w\left(t_{0}\right)\right\|_{L_{p}^{\frac{n}{p}}(\mathbb{B})}^{p} .
$$


By the continuity of $w(t)$, there exists at least one $\bar{t} \in\left(0, t_{0}\right]$ such that

$$
\left\|\Delta_{\mathbb{B}} w(\bar{t})\right\|_{L_{2}^{2}(\mathbb{B})}^{2}=b\|w(\bar{t})\|_{L_{p}^{\frac{n}{p}(\mathbb{B})}}^{p} .
$$

Let

$$
\tilde{t}=\inf \left\{\bar{t} \in\left(0, t_{0}\right]:\left\|\Delta_{\mathbb{B}} w(\bar{t})\right\|_{L_{2}^{2}(\mathbb{B})}^{2}=b\|w(\bar{t})\|_{L_{p}^{\frac{n}{p}(\mathbb{B})}}^{p}\right\} .
$$

In particular, the regularity of $w(t)$ implies that $\tilde{t} \in\left(0, t_{0}\right]$. Thus, we know

$$
\left\|\Delta_{\mathbb{B}} w(\tilde{t})\right\|_{L_{2}^{\frac{n}{2}(\mathbb{B})}}^{2}=b\|w(\tilde{t})\|_{L_{p}^{\frac{n}{p}(\mathbb{B})}}^{p}
$$

and $w(t) \in V$ for all $t \in[0, \tilde{t})$. We have two cases to consider.

First case: $\left\|\Delta_{\mathbb{B}} w(\tilde{t})\right\|_{L_{2}^{\frac{n}{2}(\mathbb{B})}}^{2}=0$.

In this case, by the continuity of $w(t)$, we have

$$
\lim _{t \rightarrow \tilde{t}^{-}}\left\|\Delta_{\mathbb{B}} w(t)\right\|_{L_{2}^{\frac{n}{2}(\mathbb{B})}}^{2}=0
$$
On the other hand, the fact that $w(t) \in V$ for all $t \in[0, \tilde{t})$ implies that $\left\|\Delta_{\mathbb{B}} w(t)\right\|_{L_{2}^{\frac{n}{2}}(\mathbb{B})}^{2} \neq 0$
and

$$
\left\|\Delta_{\mathbb{B}} w(t)\right\|_{L_{2}^{\frac{n}{2}(\mathbb{B})}}^{2}<b\|w(t)\|_{L_{p}^{\frac{n}{p}(\mathbb{B})}}^{p}, \quad t \in[0, \tilde{t}) .
$$

By Lemma 2.4, we get

$$
\|w\|_{L_{p}^{\frac{n}{p}(\mathbb{B})}}^{p} \leq C_{0}^{p}\left\|\Delta_{\mathbb{B}} w\right\|_{L_{2}^{\frac{n}{2}(\mathbb{B})}}^{p}, \quad t \in[0, \tilde{t}) .
$$

Then, by (4.4), (4.5), we have

$$
\lim _{t \rightarrow \tilde{t}^{-}}\left\|\Delta_{\mathbb{B}} w(t)\right\|_{L_{2}^{\frac{n}{2}}(\mathbb{B})}>\left(\frac{1}{b C_{0}^{p}}\right)^{\frac{1}{p-2}} .
$$

This contradicts (4.3).

Second case: $\left\|\Delta_{\mathbb{B}} w(\tilde{t})\right\|_{L_{2}^{2}(\mathbb{B})}^{2} \neq 0$.

In this case, by recalling (3.8), we know that $J(w(\tilde{t})) \geq d$. Thus, $E(\tilde{t}) \geq d$, which contradicts the fact that $E(t) \leq E(0)<d$. Hence, in either case we conclude that $w(t) \in V$ for all $t \in[0, T)$. Since

$$
J(\lambda w)=\frac{1}{2} \lambda^{2}\left\|\Delta_{\mathbb{B}} w\right\|_{L_{2}^{2}(\mathbb{B})}^{2}-\frac{b}{p} \lambda^{p}\|w\|_{L_{p}^{\frac{n}{p}(\mathbb{B})}}^{p},
$$

we obtain

$$
\frac{d}{d \lambda} J(\lambda w)=\lambda\left\|\Delta_{\mathbb{B}} w\right\|_{L_{2}^{\frac{n}{2}(\mathbb{B})}}^{2}-b \lambda^{p-1}\|w\|_{L_{p}^{\frac{n}{p}(\mathbb{B})}}^{p}
$$


and

$$
\frac{d^{2}}{d \lambda^{2}} J(\lambda w)=\left\|\Delta_{\mathbb{B}} w\right\|_{L_{2}^{2}(\mathbb{B})}^{2}-b(p-1) \lambda^{p-2}\|w\|_{L_{p}^{\frac{n}{p}}(\mathbb{B})}^{p}
$$

Let $\frac{d}{d \lambda} J(\lambda w)=0$, which implies

$$
\bar{\lambda}_{1}=0, \bar{\lambda}_{2}=\left(\frac{\left\|\Delta_{\mathbb{B}} w\right\|^{2} \frac{n}{L_{2}^{2}}(\mathbb{B})}{b\|w\|^{p}{ }_{L_{p}^{\frac{n}{p}}(\mathbb{B})}^{\frac{1}{p-2}}}\right)^{.}
$$

An elementary calculation shows

$$
\frac{d^{2}}{d \lambda^{2}} J\left(\bar{\lambda}_{1} w\right)>0, \quad \frac{d^{2}}{d \lambda^{2}} J\left(\bar{\lambda}_{2} w\right)<0 .
$$

So we have

$$
\sup _{\lambda \geq 0} J(\lambda w)=J\left(\bar{\lambda}_{2} w\right)=\frac{p-2}{2 p} \frac{\left(\left\|\Delta_{\mathbb{B}} w\right\|^{2}{ }_{L_{2}^{\frac{n}{2}}(\mathbb{B})}^{)^{\frac{p}{p-2}}}\right.}{\left(b\|w\|^{p}{ }_{L_{p}^{\frac{n}{p}}(\mathbb{B})}^{)^{\frac{2}{p-2}}}\right.} .
$$

By $I(u)<0$, we have

$$
\begin{aligned}
& d \leq \sup _{\lambda \geq 0} J(\lambda w)=J\left(\bar{\lambda}_{2} w\right)=\frac{p-2}{2 p} \frac{\left(\left\|\Delta_{\mathbb{B}} w\right\|_{L_{2}^{\frac{n}{2}}(\mathbb{B})}^{2}\right)^{\frac{p}{p-2}}}{\left(b\|w\|_{L_{p}^{\frac{n}{p}}(\mathbb{B})}^{p}\right)^{\frac{2}{p-2}}} \\
&<\frac{p-2}{2 p}\left\|\Delta_{\mathbb{B}} w\right\|_{L_{2}^{\frac{n}{2}}(\mathbb{B})}^{2} .
\end{aligned}
$$

Lemma 4.2 Let $2<p<p^{\star}$. Then there exists a positive constant $C$ depending only on $\mathbb{B}$ such that

$$
\|w\|_{L_{p}^{\frac{n}{p}}}^{s} \leq C\left(\left\|\Delta_{\mathbb{B})} w(t)\right\|_{L_{2}^{\frac{n}{2}}(\mathbb{B})}^{2}+\|w\|_{L_{p}^{\frac{n}{p}}(\mathbb{B})}^{p}\right), \quad \text { with } 2 \leq s \leq p,
$$

for any $w \in \tilde{\mathcal{H}}_{2,0}^{2, \frac{n}{2}}(\mathbb{B})$.

Proof If $\|w\|_{L_{p}^{\frac{n}{p}(\mathbb{B})}} \leq 1$, then $\|w\|_{L_{p}^{\frac{n}{p}(\mathbb{B})}}^{s} \leq\|w\|_{L_{p}^{\frac{n}{p}}{ }^{\left.\frac{n}{B}\right)}} \leq C_{0}^{2}\left\|\Delta_{\mathbb{B}} w(t)\right\|_{L_{2}^{\frac{n}{2}}(\mathbb{B})}^{2}$ by Lemma 2.4. If $\|w\|_{L_{p}^{\frac{n}{p}}(\mathbb{B})}>1$, then $\|w\|_{L_{p}^{\frac{n}{p}}(\mathbb{B})}^{s} \leq\|w\|_{L_{p}^{\frac{n}{p}}(\mathbb{B})}^{p}$. Therefore (4.7) follows.

Now we introduce the following auxiliary function:

$$
H(t)=d_{1}-E(t), \quad t \geq 0,
$$

where $d_{1}=\frac{E(0)+d}{2}>0$.

From Lemma 4.1 and Lemma 4.2, we obtain the following corollary. 
Corollary 4.1 Let the assumption of Lemma 4.2 hold. Then we have

$$
\|w\|_{L_{p}^{\frac{n}{p}(\mathbb{B})}}^{s} \leq C\left(|H(t)|+\left\|w_{t}\right\|_{L_{2}^{\frac{n}{2}}(\mathbb{B})}^{2}+\|w\|_{L_{p}^{\frac{n}{p}(\mathbb{B})}}^{p}\right), \quad \text { with } 2 \leq s \leq p
$$

for any $w \in \tilde{\mathcal{H}}_{2,0}^{2, \frac{n}{2}}(\mathbb{B})$.

Theorem 4.1 Suppose that $2<p<p^{\star}$ and $p>m \geq 2, w_{1} \in L_{2}^{\frac{n}{2}}(\mathbb{B}), w_{0} \in V$. If one of the following is satisfied:

(1) $0 \leq E(0)<d$ and $\left\|\Delta_{\mathbb{B}} w_{0}\right\|_{L_{2}^{\frac{n}{2}(\mathbb{B})}}>\lambda_{1}$;

(2) $E(0)<0$,

then the local solution w of problem (1.7)-(1.9) blows up in finite time; that is, the maximum existence time $T_{\max }$ of $w$ is finite and

$$
\lim _{T \rightarrow T_{\max }^{-}}\left[\left\|\Delta_{\mathbb{B}} w\right\|_{L_{2}^{2}(\mathbb{B})}^{2}+\|w\|_{L_{p}^{\frac{n}{p}(\mathbb{B})}}^{p}+\left\|w_{t}\right\|_{L_{2}^{\frac{n}{2}(\mathbb{B})}}^{2}\right]=+\infty
$$

Moreover, the lifespan $T_{\max }$ is estimated by $0<T_{\max } \leq \frac{1-\alpha}{\Gamma \alpha[L(0)]^{\alpha /(1-\alpha)}}$, here $L(0)$ and $\Gamma$ are given in (4.30) and (4.36) respectively. $\alpha$ is a constant given in (4.26).

Proof (1) For $0 \leq E(0)<d$, from (4.8), it follows that

$$
H^{\prime}(t)=-E^{\prime}(t)=k_{2} \int_{\mathbb{B}}\left|\nabla_{\mathbb{B}} w_{t}\right|^{2} \frac{d x_{1}}{x_{1}} d x^{\prime}+a \int_{\mathbb{B}}\left|w_{t}\right|^{m} \frac{d x_{1}}{x_{1}} d x^{\prime} \geq 0 .
$$

Thus, we have

$$
H(t) \geq H(0)=d_{1}-E(0)>0, \quad t \geq 0 .
$$

Let

$$
A(t)=\int_{\mathbb{B}} w(t) w_{t}(t) \frac{d x_{1}}{x_{1}} d x^{\prime}
$$

By differentiating (4.12) and using (1.7), (4.8), we obtain

$$
\begin{aligned}
A^{\prime}(t) & \\
= & \int_{\mathbb{B}}\left|w_{t}\right|^{2} \frac{d x_{1}}{x_{1}} d x^{\prime}+\int_{\mathbb{B}} w w_{t t} \frac{d x_{1}}{x_{1}} d x^{\prime} \\
= & \int_{\mathbb{B}}\left|w_{t}\right|^{2} \frac{d x_{1}}{x_{1}} d x^{\prime}+\int_{\mathbb{B}} w\left[-\Delta_{\mathbb{B}}^{2} w+k_{2} \Delta_{\mathbb{B}} w_{t}-a w_{t}\left|w_{t}\right|^{m-2}\right. \\
& \left.+b w|w|^{p-2}\right] \frac{d x_{1}}{x_{1}} d x^{\prime} \\
= & \left\|w_{t}\right\|_{L_{2}^{2}}^{2}{ }_{L_{(\mathbb{B})}}-\left\|\Delta_{\mathbb{B}} w\right\|_{L_{2}^{2}}^{2}{ }^{\frac{n}{2}}-k_{2} \int_{\mathbb{B}} \nabla_{\mathbb{B}} w \cdot \nabla_{\mathbb{B}} w_{t} \frac{d x_{1}}{x_{1}} d x^{\prime} \\
& +b\|w\|^{p}{ }_{L_{p} \frac{n}{p}(\mathbb{B})}-a \int_{\mathbb{B}} w w_{t}\left|u_{t}\right|^{m-2} \frac{d x_{1}}{x_{1}} d x^{\prime}
\end{aligned}
$$




$$
\begin{aligned}
= & \left(1+\frac{p}{2}\right)\left\|w_{t}\right\|_{L_{2}^{\frac{n}{2}}}^{2}+\left(\frac{p}{2}-1\right)\left\|\Delta_{\mathbb{B}} w\right\|_{L_{2}^{\frac{n}{2}(\mathbb{B})}}^{2} \\
& -a \int_{\mathbb{B}} w w_{t}\left|w_{t}\right|^{m-2} \frac{d x_{1}}{x_{1}} d x^{\prime}-k_{2} \int_{\mathbb{B}} \nabla_{\mathbb{B}} w \cdot \nabla_{\mathbb{B}} w_{t} \frac{d x_{1}}{x_{1}} d x^{\prime} \\
& +p H(t)-p d_{1} .
\end{aligned}
$$

Moreover,

$$
\begin{aligned}
& \left(\frac{p}{2}-1\right)\left\|\Delta_{\mathbb{B}} w\right\|_{L_{2}^{\frac{n}{2}(\mathbb{B})}}^{2}-p d_{1} \\
& \quad=\left(\frac{p}{2}-1\right) \frac{\lambda_{2}^{2}-\lambda_{1}^{2}}{\lambda_{2}^{2}}\left\|\Delta_{\mathbb{B}} w\right\|_{L_{2}^{\frac{n}{2}(\mathbb{B})}}^{2}+\left(\frac{p}{2}-1\right) \lambda_{1}^{2} \frac{\left\|\Delta_{\mathbb{B}} w\right\|^{2}{ }_{2}^{\frac{n}{2}(\mathbb{B})}}{\lambda_{2}^{2}}-p d_{1} \\
& \quad \geq c_{1}\left\|\Delta_{\mathbb{B}} w\right\|_{L_{2}^{\frac{n}{2}(\mathbb{B})}}^{2}+c_{2},
\end{aligned}
$$

where $\lambda_{2}$ is given in Lemma 3.2, $c_{1}=\left(\frac{p}{2}-1\right) \frac{\lambda_{2}^{2}-\lambda_{1}^{2}}{\lambda_{2}^{2}}$ and $c_{2}=\left(\frac{p}{2}-1\right) \lambda_{1}^{2}-p d_{1}$. By Lemma 3.2(ii), we have $c_{1}>0$, and by (3.7), we see that

$$
\begin{aligned}
c_{2} & =\left(\frac{p}{2}-1\right) \lambda_{1}^{2}-p d_{1} \\
& =\left(\frac{p}{2}-1\right) \lambda_{1}^{2}-\frac{p(d+E(0))}{2} \\
& =p d-\frac{p(d+E(0))}{2} \\
& =\frac{p(d-E(0))}{2}>0 .
\end{aligned}
$$

Thus, by (4.13)-(4.15), we arrive at

$$
\begin{aligned}
A^{\prime}(t)> & \left(1+\frac{p}{2}\right)\left\|w_{t}\right\|_{L_{2}^{\frac{n}{2}}}^{2}+c_{1}\left\|\Delta_{\mathbb{B}} w\right\|_{L_{2}^{\frac{n}{2}}(\mathbb{B})}^{2}-a \int_{\mathbb{B}} w w_{t}\left|w_{t}\right|^{m-2} \frac{d x_{1}}{x_{1}} d x^{\prime} \\
& -k_{2} \int_{\mathbb{B}} \nabla_{\mathbb{B}} w \cdot \nabla_{\mathbb{B}} w_{t} \frac{d x_{1}}{x_{1}} d x^{\prime}+p H(t) .
\end{aligned}
$$

We estimate the right-hand side of the above equation. By Hölder's inequality and the inequality $\|w\|_{L_{m}^{\frac{n}{m}}(\mathbb{B})} \leq C\|w\|_{L_{p}^{\frac{n}{p}}(\mathbb{B})}$, we obtain

$$
\begin{aligned}
\left.\left|\int_{\mathbb{B}} w w_{t}\right| w_{t}\right|^{m-2} \frac{d x_{1}}{x_{1}} d x^{\prime} \mid & \leq\|w\|_{L_{m}^{\frac{n}{m}(\mathbb{B})}}\left\|w_{t}\right\|_{L_{m}^{\frac{n}{m}}(\mathbb{B})}^{m-1} \\
& \leq C\|w\|_{L_{p}^{\frac{n}{p}}(\mathbb{B})}\left\|w_{t}\right\|_{L_{m}^{m}(\mathbb{B})}^{m-1} \\
& =C\|w\|_{L_{p}^{\frac{n}{m}}}^{1-\frac{p}{m}}\|w\|_{L_{p}^{\frac{n}{p}}}^{\frac{p}{m}}\left\|w_{t}\right\|_{L_{m}^{m}}^{m-1}(\mathbb{B})
\end{aligned}
$$


Note that from (4.8) and (4.2) we get

$$
\begin{aligned}
H(t)= & d_{1}-E(t) \\
< & d-\frac{1}{2}\left\|w_{t}\right\|_{L_{2}^{\frac{n}{2}}(\mathbb{B})}^{2}-\frac{1}{2}\left\|\Delta_{\mathbb{B}} w\right\|_{L_{2}^{\frac{n}{2}}(\mathbb{B})}^{2}+\frac{b}{p}\|w\|_{L_{p}^{\frac{n}{p}}(\mathbb{B})}^{p} \\
< & \frac{p-2}{2 p}\left\|\Delta_{\mathbb{B}} w\right\|_{L_{2}^{\frac{n}{2}}(\mathbb{B})}^{2}-\frac{1}{2}\left\|w_{t}\right\|_{L_{2}^{\frac{n}{2}}(\mathbb{B})}^{2}-\frac{1}{2}\left\|\Delta_{\mathbb{B}} w\right\|_{L_{2}^{\frac{n}{2}}(\mathbb{B})}^{2} \\
& +\frac{b}{p}\|w\|_{L_{p}^{\frac{n}{p}}(\mathbb{B})}^{p} \\
\leq & \frac{b}{p}\|w\|_{L_{p}^{\frac{n}{p}}(\mathbb{B})}^{p} .
\end{aligned}
$$

Thus, by (4.11) and (4.18), we see that

$$
0<H(0) \leq H(t)<\frac{b}{p}\|w\|_{L_{p}^{\frac{n}{p}}(\mathbb{B})}^{p}, \quad t \geq 0
$$

Then, using (4.19), we have from (4.17) that

$$
\left.\left|\int_{\mathbb{B}} w w_{t}\right| u_{t}\right|^{m-2} \frac{d x_{1}}{x_{1}} d x^{\prime} \mid \leq C\left(\frac{p}{b} H(t)\right)^{\frac{1}{p}\left(1-\frac{p}{m}\right)}\|w\|_{L_{p}^{\frac{n}{p}}}^{\frac{p}{m}}\left\|w_{t}\right\|_{L_{m}^{\frac{n}{m}}(\mathbb{B})}^{m-1}
$$

Hence, by Young's inequality and (4.10), we obtain

$$
\begin{aligned}
& \left.a\left|\int_{\mathbb{B}} w w_{t}\right| w_{t}\right|^{m-2} \frac{d x_{1}}{x_{1}} d x^{\prime} \mid \\
& \quad \leq c_{3} H(t)^{-\alpha^{*}}\left(\frac{a \theta^{m}}{m}\|w\|_{L_{p}^{\frac{n}{p}}(\mathbb{B})}^{p}+\frac{a(m-1)}{m} \theta^{-m /(m-1)}\left\|w_{t}\right\|_{L_{m}^{\frac{n}{m}}(\mathbb{B})}^{m}\right) \\
& \quad \leq c_{4} H(t)^{-\alpha^{*}}\left(\theta^{m}\|w\|_{L_{p}^{\frac{n}{p}}}^{p}+a \theta^{-m /(m-1)}\left\|w_{t}\right\|_{L_{m}^{\frac{n}{m}}(\mathbb{B})}^{m}\right),
\end{aligned}
$$

where $c_{3}=C\left(\frac{p}{b}\right)^{\frac{1}{p}-\frac{1}{m}}, \alpha^{*}=\frac{1}{m}-\frac{1}{p}>0, \theta>0$, and $c_{4}=c_{3} \max \left\{\frac{a}{m}, \frac{m-1}{m}\right\}$.

Letting $0<\alpha<\alpha^{*}$ and by (4.19), we see that

$$
\begin{aligned}
& \left.a\left|\int_{\mathbb{B}} w w_{t}\right| w_{t}\right|^{m-2} \frac{d x_{1}}{x_{1}} d x^{\prime} \mid \\
& \quad \leq c_{4}\left[\theta^{m} H(0)^{-\alpha^{*}}\|w\|^{p_{L_{p}^{p}}^{\frac{n}{p}}(\mathbb{B})}+\theta^{-m /(m-1)} H(t)^{-\alpha^{*}} H^{\prime}(t)\right] \\
& \leq c_{4}\left[\theta^{m} H(0)^{-\alpha^{*}}\|w\|_{L_{p}^{\frac{n}{p}(\mathbb{B})}}^{p}+\theta^{-m /(m-1)} H(0)^{\alpha-\alpha^{*}} H(t)^{-\alpha} H^{\prime}(t)\right] .
\end{aligned}
$$

Using Young's inequality again, we obtain

$$
\begin{aligned}
\int_{\mathbb{B}} \nabla_{\mathbb{B}} w \cdot \nabla_{\mathbb{B}} w_{t} \frac{d x_{1}}{x_{1}} d x^{\prime} \\
=-\int_{\mathbb{B}} \Delta_{\mathbb{B}} w \cdot w_{t} \frac{d x_{1}}{x_{1}} d x^{\prime}
\end{aligned}
$$




$$
\begin{aligned}
& \leq\left|\int_{\mathbb{B}} \Delta_{\mathbb{B}} w \cdot w_{t} \frac{d x_{1}}{x_{1}} d x^{\prime}\right| \\
& \leq \frac{1}{2}\left(\left\|\Delta_{\mathbb{B}} w\right\|_{L_{2}^{\frac{n}{2}(\mathbb{B})}}^{2}+\left\|w_{t}\right\|_{L_{2}^{\frac{n}{2}}(\mathbb{B})}^{2}\right) .
\end{aligned}
$$

Then (4.16) becomes

$$
\begin{aligned}
& A^{\prime}(t) \\
& >\left(1+\frac{p}{2}\right)\left\|w_{t}\right\|_{L_{2}^{2}(\mathbb{B})}^{2}+c_{1}\left\|\Delta_{\mathbb{B}} w\right\|_{L_{2}^{\frac{n}{2}}(\mathbb{B})}^{2}-c_{4} \theta^{m} H(0)^{-\alpha^{*}}\|w\|_{L_{p}^{\frac{n}{p}}(\mathbb{B})}^{p} \\
& \quad-c_{4} \theta^{-m /(m-1)} H(0)^{\alpha-\alpha^{*}} H(t)^{-\alpha} H^{\prime}(t)-\frac{k_{2}}{2}\left\|\Delta_{\mathbb{B}} w\right\|_{L_{2}^{\frac{n}{2}}(\mathbb{B})}^{2} \\
& \quad-\frac{k_{2}}{2}\left\|w_{t}\right\|_{L_{2}^{\frac{n}{2}}(\mathbb{B})}^{2}+p H(t) .
\end{aligned}
$$

Now, we define

$$
L(t)=H^{1-\alpha}(t)+\varepsilon A(t), \quad t \geq 0,
$$

where $\varepsilon$ is small to be specified later and

$$
0<\alpha \leq \frac{p-2}{2 p}
$$

By differentiating (4.25), by Lemma 2.2 and (4.24), we see that

$$
\begin{aligned}
L^{\prime}(t)= & (1-\alpha) H^{-\alpha}(t) H^{\prime}(t)+\varepsilon A^{\prime}(t) \\
> & (1-\alpha) H^{-\alpha}(t) H^{\prime}(t)+\varepsilon\left[\left(1+\frac{p}{2}-\frac{k_{2}}{2}\right)\left\|w_{t}\right\|_{L_{2}^{\frac{n}{2}}(\mathbb{B})}^{2}\right. \\
& +\left(c_{1}-\frac{k_{2}}{2}\right)\left\|\Delta_{\mathbb{B}} w\right\|_{L_{2}^{\frac{n}{2}}(\mathbb{B})}^{2}-c_{4} \theta^{m} H(0)^{-\alpha^{*}}\|w\|_{L_{p}^{\frac{n}{p}}(\mathbb{B})}^{p} \\
& \left.-c_{4} \theta^{-m /(m-1)} H(0)^{\alpha-\alpha^{*}} H(t)^{-\alpha} H^{\prime}(t)+p H(t)\right] .
\end{aligned}
$$

Letting

$$
a_{1}=\min \left\{\frac{p}{2}, c_{1}-\frac{k_{2}}{2}, 1+\frac{p}{2}-\frac{k_{2}}{2}\right\}>0
$$

and decomposing $\varepsilon p H(t)$ in (4.27) by

$$
\varepsilon p H(t)=2 a_{1} \varepsilon H(t)+\left(p-2 a_{1}\right) \varepsilon H(t) .
$$


Thus, by (4.8) and (3.3), we obtain

$$
\begin{aligned}
& L^{\prime}(t)>\left(1-\alpha-c_{4} \varepsilon \theta^{-m /(m-1)} H(0)^{\alpha-\alpha^{*}}\right) H^{-\alpha}(t) H^{\prime}(t) \\
& +\varepsilon\left(1+\frac{p}{2}-\frac{k_{2}}{2}\right)\left\|w_{t}\right\|_{L_{2}^{2}}^{2}{ }_{(\mathbb{B})}+\varepsilon\left(c_{1}-\frac{k_{2}}{2}\right)\left\|\Delta_{\mathbb{B}} w\right\|_{L_{2}^{2}{ }^{\frac{n}{2}}}^{2} \\
& -c_{4} \varepsilon \theta^{m} H(0)^{-\alpha^{*}}\|w\|_{L_{p}^{\frac{n}{p}}(\mathbb{B})}^{p}+2 a_{1} \varepsilon\left(-\frac{1}{2}\left\|w_{t}\right\|_{L_{2}^{\frac{n}{2}}(\mathbb{B})}^{2}\right. \\
& \left.-\frac{1}{2}\left\|\Delta_{\mathbb{B}} w\right\|_{L_{2}^{\frac{n}{2}(\mathbb{B})}}^{2}+\frac{b}{p}\|w\|_{L_{p}^{\frac{n}{p}(\mathbb{B})}}^{p}\right)+\left(p-2 a_{1}\right) \varepsilon H(t) \\
& =\left(1-\alpha-c_{4} \varepsilon \theta^{-m /(m-1)} H(0)^{\alpha-\alpha^{*}}\right) H^{-\alpha}(t) H^{\prime}(t) \\
& +\varepsilon\left(1+\frac{p}{2}-\frac{k_{2}}{2}-a_{1}\right)\left\|w_{t}\right\|_{L_{2}^{\frac{n}{2}}(\mathbb{B})}^{2} \\
& +\varepsilon\left(c_{1}-\frac{k_{2}}{2}-a_{1}\right)\left\|\Delta_{\mathbb{B}} w\right\|_{L_{2}^{2}(\mathbb{B})}^{2} \\
& +\varepsilon\left[\frac{2 a_{1} b}{p}-c_{4} \theta^{m} H(0)^{-\alpha^{*}}\right]\|w\|_{L_{p}^{\frac{n}{p}}}^{p}(\mathbb{B}) \\
& +\left(p-2 a_{1}\right) \varepsilon H(t) \text {. }
\end{aligned}
$$

Now, we choose $\theta>0$ small such that

$$
\frac{2 a_{1} b}{p}-c_{4} \theta^{m} H(0)^{-\alpha^{*}} \geq \frac{a_{1} b}{2 p}
$$

and we pick $\varepsilon$ small enough so that

$$
1-\alpha-c_{4} \varepsilon \theta^{-m /(m-1)} H(0)^{\alpha-\alpha^{*}} \geq 0 .
$$

Then (4.28) becomes

$$
L^{\prime}(t)>c_{5} \varepsilon\left(\left\|w_{t}\right\|_{L_{2}^{\frac{n}{2}}(\mathbb{B})}^{2}+\left\|\Delta_{\mathbb{B}} w\right\|_{L_{2}^{\frac{n}{2}}(\mathbb{B})}^{2}+\|w\|_{L_{p}^{p}(\mathbb{B})}^{p}+H(t)\right),
$$

here $c_{5}=\min \left\{\frac{a_{1} b}{2 p}, c_{1}-\frac{k_{2}}{2}-a_{1}, 1+\frac{p}{2}-\frac{k_{2}}{2}-a_{1}, p-2 a_{1}\right\}$. Thus $L(t)$ is a nondecreasing function on $t \geq 0$, and we take $\varepsilon$ small enough such that

$$
L(0)=H^{1-\alpha}(0)+\varepsilon \int_{\mathbb{B}} w_{0} w_{1} \frac{d x_{1}}{x_{1}} d x^{\prime}>0 .
$$

Hence, we have

$$
L(t)>0, \quad \forall t \geq 0 .
$$

Next we estimate the second term in (4.25) as follows:

$$
\left|\int_{\mathbb{B}} w w_{t} \frac{d x_{1}}{x_{1}} d x^{\prime}\right| \leq\|w\|_{L_{2}^{\frac{n}{2}}(\mathbb{B})}\left\|w_{t}\right\|_{L_{2}^{\frac{n}{2}}(\mathbb{B})} \leq C\|w\|_{L_{p}^{\frac{n}{p}}(\mathbb{B})}\left\|w_{t}\right\|_{L_{2}^{\frac{n}{2}}(\mathbb{B})} .
$$


So we have

$$
\left|\int_{\mathbb{B}} w w_{t} \frac{d x_{1}}{x_{1}} d x^{\prime}\right|^{1 /(1-\alpha)} \leq C\|w\|_{L_{p}^{p}(\mathbb{B})}^{1 /(1-\alpha)}\left\|w_{t}\right\|_{L_{2}^{\frac{n}{2}(\mathbb{B})}}^{1 /(1-\alpha)}
$$

Again Young's inequality gives

$$
\left|\int_{\mathbb{B}} w w_{t} \frac{d x_{1}}{x_{1}} d x^{\prime}\right|^{1 /(1-\alpha)} \leq C\left[\|w\|_{L_{p}^{\frac{n}{p}(\mathbb{B})}}^{\mu_{1} /(1-\alpha)}+\left\|w_{t}\right\|_{L_{2}^{2}(\mathbb{B})}^{\mu_{2} /(1-\alpha)}\right] .
$$

We take $\mu_{1}=\frac{2(1-\alpha)}{1-2 \alpha}, \mu_{2}=2(1-\alpha)$ to get $\mu_{1} /(1-\alpha)=2 /(1-2 \alpha) \leq p$ by condition (4.26). Therefore (4.32) becomes

$$
\left|\int_{\mathbb{B}} w w_{t} \frac{d x_{1}}{x_{1}} d x^{\prime}\right|^{1 /(1-\alpha)} \leq C\left[\|w\|_{L_{p}^{\frac{n}{p}(\mathbb{B})}}^{s}+\left\|w_{t}\right\|_{L_{2}^{2}(\mathbb{B})}^{2}\right]
$$

where $s=2 /(1-2 \alpha) \leq p$. By using Corollary 4.1, we obtain

$$
\left|\int_{\mathbb{B}} w w_{t} \frac{d x_{1}}{x_{1}} d x^{\prime}\right|^{1 /(1-\alpha)} \leq C\left[H(t)+\|w\|_{L_{p}^{\frac{n}{p}}(\mathbb{B})}^{p}+\left\|w_{t}\right\|_{L_{2}^{\frac{n}{2}}(\mathbb{B})}^{2}\right], \quad \forall t \geq 0 .
$$

Consequently, we have

$$
\begin{aligned}
L^{1 /(1-\alpha)}(t) & =\left(H^{1-\alpha}(t)+\varepsilon \int_{\mathbb{B}} w w_{t} \frac{d x_{1}}{x_{1}} d x^{\prime}\right)^{1 /(1-\alpha)} \\
& \leq 2^{\alpha /(1-\alpha)}\left(H(t)+\left|\int_{\mathbb{B}} w w_{t} \frac{d x_{1}}{x_{1}} d x^{\prime}\right|^{1 /(1-\alpha)}\right) \\
& \leq C\left[H(t)+\left\|\Delta_{\mathbb{B}} w\right\|_{L_{2}^{\frac{n}{2}(\mathbb{B})}}^{2}+\|w\|_{L_{p}^{p}(\mathbb{B})}^{p}+\left\|w_{t}\right\|_{L_{2}^{\frac{n}{2}(\mathbb{B})}}^{2}\right] .
\end{aligned}
$$

We then combine (4.29) and (4.35) to arrive at

$$
L^{\prime}(t) \geq \Gamma L^{1 /(1-\alpha)}(t)
$$

where $\Gamma$ is a constant dependent on $C, c_{3}$ and $\varepsilon$ only (and hence is independent of the solution $w)$. A simple integration of $(4.36)$ over $(0, t)$ then yields

$$
L^{\alpha /(1-\alpha)}(t) \geq \frac{1}{L^{-\alpha /(1-\alpha)}(0)-\Gamma t \alpha /(1-\alpha)} .
$$

Since $L(0)>0$, (4.37) shows that $L(t)$ becomes infinite in a finite time $T_{\max } \leq T^{*}=$ $\frac{1-\alpha}{\Gamma \alpha[L(0))^{\alpha /(1-\alpha)}}$.

(2) For $E(0)<0$, we set

$$
H(t)=-E(t)
$$

instead of (4.8). Then, applying the same arguments as in part (1), we have the result. 
Theorem 4.2 Under the assumption of Theorem 4.1, let $w(x, t)$ be a blow-up solution of problem (1.7)-(1.9). Then a lower bound T for the lifespan $t^{\star}$ of $w$ is given by

$$
T:=\int_{\phi(0)}^{+\infty} \frac{d s}{\bar{c}_{2} S^{\frac{\alpha(p-1)}{p(\alpha-1)}}+\bar{c}_{3}} \leq t^{\star}
$$

with

$$
\phi(0)=\frac{1}{2} \int_{\mathbb{B}}\left|w_{1}\right|^{2} \frac{d x_{1}}{x_{1}} d x^{\prime}+\frac{1}{2} \int_{\mathbb{B}}\left|\Delta_{\mathbb{B}} w_{0}\right|^{2} \frac{d x_{1}}{x_{1}} d x^{\prime}+\frac{b}{p} \int_{\mathbb{B}}\left|w_{0}\right|^{p} \frac{d x_{1}}{x_{1}} d x^{\prime},
$$

where $1<\alpha<2$ and $\bar{c}_{2}, \bar{c}_{3}$ are positive constants to be determined later.

Proof Now we want to derive a lower bound for the lifespan $t^{\star}$ of the blow-up solution. To this end, we introduce the auxiliary function

$$
\phi(t)=\frac{1}{2} \int_{\mathbb{B}}\left|w_{t}\right|^{2} \frac{d x_{1}}{x_{1}} d x^{\prime}+\frac{1}{2} \int_{\mathbb{B}}\left|\Delta_{\mathbb{B}} w\right|^{2} \frac{d x_{1}}{x_{1}} d x^{\prime}+\frac{b}{p} \int_{\mathbb{B}}|w|^{p} \frac{d x_{1}}{x_{1}} d x^{\prime}
$$

and compute a value $T>0$ such that $\phi(t)$ remains bounded for $t \in[0, T]$. Clearly, $T$ is a lower bound for $t^{\star}$. Differentiating (4.39) and making use of the second Green's formula, we obtain in view of (1.7)

$$
\begin{aligned}
\phi^{\prime}(t)= & \int_{\mathbb{B}} w_{t} w_{t t} \frac{d x_{1}}{x_{1}} d x^{\prime}+\int_{\mathbb{B}} \Delta_{\mathbb{B}} w \cdot \Delta_{\mathbb{B}} w_{t} \frac{d x_{1}}{x_{1}} d x^{\prime} \\
& +b \int_{\mathbb{B}}|w|^{p-2} w w_{t} \frac{d x_{1}}{x_{1}} d x^{\prime} \\
= & \int_{\mathbb{B}} w_{t}\left[2 b|w|^{p-2} w-a w_{t}\left|u_{t}\right|^{m-2}+k_{2} \Delta_{\mathbb{B}} w_{t}\right] \frac{d x_{1}}{x_{1}} d x^{\prime} \\
= & 2 b \int_{\mathbb{B}}|w|^{p-2} w w_{t} \frac{d x_{1}}{x_{1}} d x^{\prime}-a \int_{\mathbb{B}}\left|w_{t}\right|^{m} \frac{d x_{1}}{x_{1}} d x^{\prime} \\
& -k_{2} \int_{\mathbb{B}}\left|\nabla_{\mathbb{B}} w_{t}\right|^{2} \frac{d x_{1}}{x_{1}} d x^{\prime} \\
\leq & 2 b \int_{\mathbb{B}}\left|w_{t}\right||w|^{p-1} \frac{d x_{1}}{x_{1}} d x^{\prime}-k_{2} \int_{\mathbb{B}}\left|\nabla_{\mathbb{B}} w_{t}\right|^{2} \frac{d x_{1}}{x_{1}} d x^{\prime} .
\end{aligned}
$$

Now we make use of Hölder's inequality to the first term on the right-hand side of (4.40) to obtain

$$
\begin{aligned}
\int_{\mathbb{B}}\left|w_{t} \| w\right|^{p-1} \frac{d x_{1}}{x_{1}} d x^{\prime} & \leq\left(\int_{\mathbb{B}}|w|^{p} \frac{d x_{1}}{x_{1}} d x^{\prime}\right)^{\frac{p-1}{p}}\left(\int_{\mathbb{B}}\left|w_{t}\right|^{p} \frac{d x_{1}}{x_{1}} d x^{\prime}\right)^{\frac{1}{p}} \\
& =\|w\|_{L_{p}^{p-1}}^{p-1}\left\|w_{t}\right\|_{L_{p}^{\frac{n}{p}}} .
\end{aligned}
$$

Then

$$
\phi^{\prime}(t) \leq 2 b\|w\|_{L_{p}^{\frac{n}{p}}}^{p-1}\left\|w_{t}\right\|_{L_{p}^{\frac{n}{p}}}-k_{2} \int_{\mathbb{B}}\left|\nabla_{\mathbb{B}} w_{t}\right|^{2} \frac{d x_{1}}{x_{1}} d x^{\prime} .
$$


In the rest of the proof, we apply Young's inequality to the first term on the right-hand side of (4.42) with exponents $\alpha$ and $\frac{\alpha}{\alpha-1}$, where $1<\alpha<2$ is a constant. Thus we obtain

$$
\begin{aligned}
2 b\|w\|_{L_{p}^{\frac{n}{p}}}^{p-1}\left\|w_{t}\right\|_{L_{p}^{\frac{n}{p}}} & \leq \bar{c}_{1}\|w\|_{L_{p}^{\frac{\alpha-1}{p}}}^{\frac{\alpha(p-1)}{{ }^{\frac{1}{p}}}}+\left\|w_{t}\right\|_{L_{p}^{\frac{n}{p}}}^{\alpha} \\
& \leq \bar{c}_{2} \phi(t)^{\frac{\alpha(p-1)}{p(\alpha-1)}}+c_{\star}^{\alpha}\left\|\nabla_{\mathbb{B}} w_{t}\right\|_{L_{2}^{\alpha}}^{\alpha}
\end{aligned}
$$

with $\bar{c}_{1}=(2 b)^{\frac{\alpha}{\alpha-1}} \alpha^{-\frac{1}{\alpha-1}} \frac{\alpha-1}{\alpha}$ and $\bar{c}_{2}=\bar{c}_{1}\left(\frac{p}{b}\right)^{\frac{\alpha(p-1)}{p(\alpha-1)}}$. Because of $\alpha<2$, we can use Young's inequality with exponents $\frac{2}{\alpha}$ and $\frac{2}{2-\alpha}$ to have

$$
c_{\star}^{\alpha}\left\|\nabla_{\mathbb{B}} w_{t}\right\|_{L_{2}^{\frac{n}{2}}}^{\alpha} \leq k_{2}\left\|\nabla_{\mathbb{B}} w_{t}\right\|_{L_{2}^{\frac{n}{2}}}^{2}+\bar{c}_{3}
$$

with $\bar{c}_{3}=c_{\star}^{\frac{2 \alpha}{2-\alpha}}\left(\frac{\alpha}{2 k_{2}}\right)^{\frac{\alpha}{2-\alpha}} \frac{2-\alpha}{2}$. Inserting this in (4.43) yields

$$
2 b\|w\|_{L_{p}^{\frac{n}{p}}}^{p-1}\left\|w_{t}\right\|_{L_{p}^{\frac{n}{p}}} \leq \bar{c}_{2} \phi(t)^{\frac{\alpha(p-1)}{p(\alpha-1)}}+k_{2}\left\|\nabla_{\mathbb{B}} w_{t}\right\|_{L_{2}^{\frac{n}{2}}}^{2}+\bar{c}_{3} .
$$

Inequality (4.44) along with (4.42) implies that

$$
\phi^{\prime}(t) \leq \bar{c}_{2} \phi(t)^{\frac{\alpha(p-1)}{p(\alpha-1)}}+\bar{c}_{3} .
$$

Then

$$
\frac{d \phi}{\bar{c}_{2} \phi(t)^{\frac{\alpha(p-1)}{p(\alpha-1)}}+\bar{c}_{3}} \leq d t .
$$

Integrating (4.46) from 0 to $t^{\star}$, we obtain

$$
\int_{\phi(0)}^{\phi(t)} \frac{d s}{\bar{c}_{2} S^{\frac{\alpha(p-1)}{p(\alpha-1)}}+\bar{c}_{3}} \leq t^{\star} .
$$

Thus, we obtain the desired result.

In the following theorem, by means of a first order differential inequality technique, we obtain a lower bound for the blow-up time which is different from (4.38).

Theorem 4.3 Suppose that the conditions of Theorem 4.1 hold. Let $w(x, t)$ be a blow-up solution of problem (1.7)-(1.9). Then a lower bound $\tilde{T}$ for the lifespan $t^{\star}$ of $w$ is given by

$$
\tilde{T}:=\left\{(p-2) b \kappa^{\frac{1}{2}}(\psi(0))^{\frac{p-2}{2}}\right\}^{-1}<t^{\star},
$$

with

$$
\psi(0)=\int_{\mathbb{B}}\left|w_{1}\right|^{2} \frac{d x_{1}}{x_{1}} d x^{\prime}+\int_{\mathbb{B}}\left|\Delta_{\mathbb{B}} w_{0}\right|^{2} \frac{d x_{1}}{x_{1}} d x^{\prime},
$$

where $\kappa=C_{0}^{2(p-1)}$. 
Proof We introduce the auxiliary function

$$
\psi(t)=\int_{\mathbb{B}}\left|w_{t}\right|^{2} \frac{d x_{1}}{x_{1}} d x^{\prime}+\int_{\mathbb{B}}\left|\Delta_{\mathbb{B}} w\right|^{2} \frac{d x_{1}}{x_{1}} d x^{\prime}
$$

and compute a value $\tilde{T}>0$ such that $\psi(t)$ remains bounded for $t \in[0, \tilde{T}]$. Clearly $\tilde{T}$ is a lower bound for $t^{\star}$. Differentiating (4.49) and making use of the second Green's formula, we obtain in view of (1.7)

$$
\begin{aligned}
\psi^{\prime}(t)= & 2 \int_{\mathbb{B}} w_{t} w_{t t} \frac{d x_{1}}{x_{1}} d x^{\prime}+2 \int_{\mathbb{B}} \Delta_{\mathbb{B}} w \cdot \Delta_{\mathbb{B}} w_{t} \frac{d x_{1}}{x_{1}} d x^{\prime} \\
= & 2 \int_{\mathbb{B}} w_{t}\left[w_{t t}+\Delta_{\mathbb{B}}^{2} w\right] \frac{d x_{1}}{x_{1}} d x^{\prime} \\
= & 2 b \int_{\mathbb{B}}|w|^{p-2} w w_{t} \frac{d x_{1}}{x_{1}} d x^{\prime}-2 a \int_{\mathbb{B}}\left|w_{t}\right|^{m} \frac{d x_{1}}{x_{1}} d x^{\prime} \\
& -2 k_{2} \int_{\mathbb{B}}\left|\nabla_{\mathbb{B}} w_{t}\right|^{2} \frac{d x_{1}}{x_{1}} d x^{\prime} \\
\leq & 2 b \int_{\mathbb{B}}\left|w_{t}\right||w|^{p-1} \frac{d x_{1}}{x_{1}} d x^{\prime} .
\end{aligned}
$$

Making use of the Schwarz inequality leads to

$$
\psi^{\prime}(t) \leq 2 b\left(\int_{\mathbb{B}}\left|w_{t}\right|^{2} \frac{d x_{1}}{x_{1}} d x^{\prime} \int_{\mathbb{B}}|w|^{2(p-1)} \frac{d x_{1}}{x_{1}} d x^{\prime}\right)^{\frac{1}{2}} .
$$

Applying the Poincaré inequality, we obtain

$$
\begin{aligned}
& \int_{\mathbb{B}}|w|^{2(p-1)} \frac{d x_{1}}{x_{1}} d x^{\prime}=\|w\|^{2(p-1)} \\
& \leq C_{2(p-1)}^{2(p-1)}\left\|\Delta_{\mathbb{B}} w\right\|^{2(p-1)} \\
& L_{2}^{\frac{n}{2(p-1)}} \\
& \leq C_{0}^{2(p-1)}(\psi(t))^{p-1}
\end{aligned}
$$

Moreover, we have

$$
\int_{\mathbb{B}}\left|w_{t}\right|^{2} \frac{d x_{1}}{x_{1}} d x^{\prime}<\psi(t) .
$$

From (4.51)-(4.53), we obtain the differential inequality

$$
\psi^{\prime}(t)<2 b \kappa^{\frac{1}{2}} \psi(t)^{\frac{p}{2}}
$$

where $\kappa=C_{0}^{2(p-1)}$, then (4.54) can be rewritten as

$$
\left(\psi^{\frac{2-p}{2}}(t)\right)^{\prime}>-(p-2) b \kappa^{\frac{1}{2}} .
$$

Integrating (4.55) from 0 to $t$, we obtain

$$
\left(\psi^{\frac{2-p}{2}}(t)\right)>\psi^{\frac{2-p}{2}}(0)-(p-2) b \kappa^{\frac{1}{2}} t .
$$


Inequality (4.56) shows that $\psi(t)$ remains bounded for

$$
t<\tilde{T}:=\frac{(\psi(0))^{\frac{2-p}{2}}}{(p-2) b \kappa^{\frac{1}{2}}} .
$$

From the discussion above in Theorem 3.1 and Theorem 4.1, we immediately obtain a specifying result of the global existence and nonexistence of solutions for problem (1.7)(1.9) as follows.

Remark 4.1 Suppose that $2<p<p^{\star}, w_{1} \in L_{2}^{\frac{n}{2}}(\mathbb{B})$, and $0<E(0)<d$, then problem (1.7)-(1.9) admits a global weak solution without relation between $m$ and $p$ provided $I\left(w_{0}\right)>0$ and $w$ satisfies the assumption of Lemma 3.3; problem (1.7)-(1.9) does not admit any global solution provided $p>m \geq 2, I\left(w_{0}\right)<0$, and $\left\|\Delta_{\mathbb{B}} w_{0}\right\|_{L_{2}^{\frac{n}{2}}(\mathbb{B})}>\lambda_{1}$.

From the discussion above in Theorem 4.1 and Theorem 4.2, we give the bounds for blow-up time for problem (1.7)-(1.9) under the initial condition $I\left(w_{0}\right)<0$.

Remark 4.2 Suppose that $2<p<p^{\star}, p>m \geq 2$ and $E(0)<d, w_{1} \in L_{2}^{\frac{n}{2}}(\mathbb{B})$, then problem (1.7)-(1.9) does not admit any global solution provided $I\left(w_{0}\right)<0$. Furthermore, the corresponding upper and lower bounds of blow-up time $T_{\max }$ are given by the following form:

$$
\int_{\phi(0)}^{+\infty} \frac{d s}{\bar{c}_{2} S^{\frac{\alpha(p-1)}{p(\alpha-1)}}+\bar{c}_{3}} \leq T_{\max } \leq \frac{1-\alpha}{\Gamma \alpha[L(0)]^{\alpha /(1-\alpha)}}
$$

\section{Acknowledgements}

The authors thank the referees for valuable comments and suggestions which improved the presentation of this manuscript.

\section{Funding}

This work was supported by the NSF of China (Grant Nos. 11701116, 11801108, and 11871172).

\section{Abbreviations}

Not applicable.

Availability of data and materials

Not applicable.

Competing interests

The authors declare that they have no competing interests.

\section{Authors' contributions}

Each of the authors contributed to each part of this study equally. All authors read and approved the final vision of the manuscript.

\section{Author details}

'School of Science, Dalian Jiaotong University, Dalian, 116028, P.R. China. ${ }^{2}$ School of Mathematics and Information

Science, Guangzhou University, Guangzhou, 510006, P.R. China.

\section{Publisher's Note}

Springer Nature remains neutral with regard to jurisdictional claims in published maps and institutional affiliations.

Received: 2 May 2020 Accepted: 12 August 2020 Published online: 20 August 2020

\section{References}

1. Reed, M., Simon, B.: Methods of Modern Mathematical Physics. Scattering Theory, vol. III. Academic Press, New York (1979)

2. Zauderer, E.: Partial Differential Equations of Applied Mathematics, 2nd edn. Pure and Applied Mathematics. A Wiley-Interscience Publication. Wiley, New York (1989) 
3. Li, G., Sun, Y.N., Liu, W.J.: Global existence and blow-up of solutions for a strongly damped Petrovsky system with nonlinear damping. Appl. Anal. 3, 575-586 (2012)

4. Pikin, E., Polat, N.: On the decay of solutions for a nonlinear Petrovsky equation. Math. Sci. Lett. 1, 43-47 (2013)

5. You, Y.C.: Energy decay and exact controllability for the Petrovsky equation in a bounded domain. Adv. Appl. Math. $11,372-388(1990)$

6. Amroun, N.E., Benaissa, A.: Solutions to a Petrovsky equation with general nonlinear dissipation and source term. Georgian Math. J. 13, 397-410 (2006)

7. Zhou, Y: Global existence and nonexistence for a nonlinear wave equation with damping and source terms. Math. Nachr. 278, 1341-1358 (2005)

8. Zhou, Y.: A blow-up result for a nonlinear wave equation with damping and vanishing initial energy in $R^{\mathbb{N}}$. Appl. Math Lett. 18, 281-286 (2005)

9. Messaoudi, S.A.: Global existence and nonexistence in a system of Petrovsky. J. Math. Anal. Appl. 265, 296-308 (2002)

10. Wu, S.T., Tasi, L.Y.: On global solutions and blow-up solutions for a nonlinear damped Petrovsky system. Taiwan. J. Math. 13, 545-558 (2009)

11. Chen, W.Y., Zhou, Y: Global nonexistence for a semilinear Petrovsky equation. Nonlinear Anal. 70, 3203-3208 (2009)

12. Philippin, G.A., Vernier, P.S.: Lower bound for the lifespan of solutions for a class of fourth order wave equations. Appl. Math. Lett. 50, 141-145 (2015)

13. Baghaei, K.: Lower bounds for the blow-up time in a superlinear hyperbolic equation with linear damping term. Comput. Math. Appl. 73, 560-564 (2017)

14. Komornik, V., Patcheu, S.K.: Well-posedness and decay estimates for a Petrovsky system with internal damping. Adv. Math. Sci. Appl. 7, 245-260 (1997)

15. Messaoudi, S.A.: Global existence and decay of solutions to a system of Petrovsky. Math. Sci. Res. J. 6, 534-541 (2002)

16. Xu, R.Z., Liu, Y.C., Yu, T.: Global existence of solution for Cauchy problem of multidimensional generalized double dispersion equations. Nonlinear Anal. 71, 4977-4983 (2009)

17. Xu, R.Z., Chen, T.L., Liu, C.M., Ding, Y.H.: Global well-posedness and global attractor of fourth order semilinear parabolic equation. Math. Methods Appl. Sci. 38, 1515-1529 (2015)

18. Peng, X.M., Shang, Y.D., Zheng, X.X.: Lower bounds for the blow-up time to a nonlinear viscoelastic wave equation with strong damping. Appl. Math. Lett. 76, 66-73 (2018)

19. Chen, H., Liu, X.C., Wei, Y.W.: Existence theorem for a class of semilinear totally characteristic elliptic equations with critical cone Sobolev exponents. Ann. Glob. Anal. Geom. 39, 27-43 (2011)

20. Chen, H., Liu, X.C., Wei, Y.W.: Cone Sobolev inequality and Dirichlet problem for nonlinear elliptic equations on a manifold with conical singularities. Calc. Var. Partial Differ. Equ. 43, 463-484 (2012)

21. Chen, H., Liu, G.W.: Global existence and nonexistence for semilinear parabolic equations with conical degeneration. J. Pseudo-Differ. Oper. Appl. 3, 329-349 (2012)

22. Li, G., Yu, J.Y., Liu, W.J.: Global existence, exponential decay and finite time blow-up of solutions for a class of semilinear pseudo-parabolic equations with conical degeneration. J. Pseudo-Differ. Oper. Appl. 8, 629-660 (2017)

23. Alimohammady, M., Cattani, C., Kalleji, M.K.: Invariance and existence analysis for semilinear hyperbolic equations with damping and conical singularity. J. Math. Anal. Appl. 455, 569-591 (2017)

24. Sattinger, D.H.: On global solution of nonlinear hyperbolic equations. Arch. Ration. Mech. Anal. 30, 148-172 (1968)

25. Alimohammady, M., Kalleji, M.K.: Existence result for a class of semilinear totally characteristic hypoelliptic equations with conical degeneration. J. Funct. Anal. 265, 2331-2356 (2013)

26. Alimohammady, M., Kalleji, M.K., Karamali, G.: Global results for semilinear hyperbolic equations with damping term on manifolds with conical singularity. Math. Methods Appl. Sci. 40, 4160-4178 (2017)

27. Alimohammady, M., Jafari, A.A., Kalleji, M.K.: Multiple solutions for non-homogenous degenerate Schrödinger equations in cone Sobolev spaces. Indian J. Pure Appl. Math. 48, 133-146 (2017)

28. Di, H.F., Shang, Y.D.: Global well-posedness for a nonlocal semi-linear pseudo-parabolic equation with conical degeneration. J. Differ. Equ. 269, 4566-4597 (2020)

29. Xu, R.Z., Su, J.: Global existence and finite time blow-up for a class of semilinear pseudo-parabolic equations. J. Funct. Anal. 264, 2732-2763 (2013)

30. Lian, W., Xu, R.Z., Rădulescu, V.D., Yang, Y.B., Zhao, N.: Global well-posedness for a class of fourth order nonlinear strongly damped wave equations. Adv. Calc. Var. (2019). https://doi.org/10.1515/acv-2019-0039

31. Lian, W., Xu, R.Z:: Global well-posedness of nonlinear wave equation with weak and strong damping terms and logarithmic source term. Adv. Nonlinear Anal. 9, 613-632 (2020)

32. Schrohe, E., Seiler, J.: Ellipticity and invertibility in the cone algebra on $L_{p}$-Sobolev spaces. Integral Equ. Oper. Theory 41, 93-114 (2001) 